\title{
Almost Sure Central Limit Theorems in Stochastic Geometry
}

\author{
Giovanni Luca Torrisi* $\quad$ Emilio Leonardi ${ }^{\dagger}$
}

\begin{abstract}
We prove an almost sure central limit theorem on the Poisson space, which is perfectly tailored for stabilizing functionals emerging in stochastic geometry. As a consequence, we provide almost sure central limit theorems for $(i)$ the total edge length of the $k$-nearest neighbors random graph, (ii) the clique count in random geometric graphs, (iii) the volume of the set approximation via the Poisson-Voronoi tessellation.
\end{abstract}

Key words: almost sure limit theorem; Malliavin calculus, Poisson process, random graphs, stabilization, stochastic geometry. Mathematics Subject Classification (2010): 60F05, 60G55, 60H07, 60D05

\section{Introduction}

Let $\left\{X_{n}\right\}_{n \geq 1}$ be a sequence of real-valued random variables and $Z$ a random variable distributed according to the standard normal law. We say that $\left\{X_{n}\right\}_{n \geq 1}$ satisfies the almost sure central limit theorem (ASCLT) if

$$
\lim _{n \rightarrow \infty} \frac{1}{\log n} \sum_{k=1}^{n} \frac{1}{k} f\left(X_{k}\right)=\mathbb{E}[f(Z)], \quad \forall f \in \mathcal{C}_{b}(\mathbb{R}), \text { almost surely }
$$

i.e., the sequence of random measures

$$
\left\{\frac{1}{\log n} \sum_{k=1}^{n} \frac{1}{k} \varepsilon_{X_{k}}\right\}_{n \geq 2}
$$

converges weakly to the standard normal law, almost surely. Roughly speaking, if an ASCLT holds, then the Gaussian asymptotic behavior can be observed along individual trajectories of the process. Here $\mathcal{C}_{b}(\mathbb{R})$ denotes the family of bounded and continuous functions from $\mathbb{R}$ to $\mathbb{R}$ and $\varepsilon_{x}$ denotes the Dirac measure at $x$.

Property (1.1) should be compared with the classical notion of weak convergence of $\left\{X_{n}\right\}_{n \geq 1}$ to the random variable $Z$, which can be stated as follows:

$$
\lim _{n \rightarrow \infty} \mathbb{E}\left[f\left(X_{n}\right)\right]=\mathbb{E}[f(Z)], \quad \forall f \in \mathcal{C}_{b}(\mathbb{R}) .
$$

\footnotetext{
*Istituto per le Applicazioni del Calcolo, CNR, Roma, Italy. e-mail: giovanniluca.torrisi@cnr.it

${ }^{\dagger}$ Dipartimento di Elettronica, Politecnico di Torino, Italy. e-mail: emilio.leonardi@polito.it
} 
In (1.1) expectation, $\mathbb{E}$, is replaced by logarithmic average, $\frac{1}{\log n} \sum_{k=1}^{n} \frac{1}{k}$, while almost sure convergence is considered. Moreover, as it has been recently pointed out in [2], a simple application of Skorohod's representation theorem shows that, in contrast to (1.2), the validity of (1.1) depends on the whole sequence $\left\{X_{n}\right\}_{n \geq 1}$, and that (1.2) does not imply (1.1).

The ASCLT for the sequence $\left\{S_{n}\right\}_{n \geq 1}$, where $S_{n}:=n^{-1 / 2} \sum_{k=1}^{n} X_{k}$ and $X_{n}, n \geq 1$, are independent and identically distributed real-valued random variables with $\mathbb{E}\left[X_{1}\right]=0$ and $\mathbb{E}\left[X_{1}^{2}\right]=1$, was conjectured by Lévy [12] and proved in [5] and [19] independently. Since then ASCLTs have attracted a significant interest in the scientific community. For instance, it was proved in [4] that (basically) whenever a sequence of independent and identically distributed random variables converges in distribution, the corresponding almost sure limit theorem holds. The situation is much more complicated for dependent sequences, see e.g. [6, 13, 22]. More recently, in [3] the authors proved ASCLTs for sequences of functionals of general Gaussian fields, with applications to stationary Gaussian sequences. The main idea was to employ the Malliavin calculus on the Wiener space in order to check the conditions of the so-called Ibragimov-Lifshits criterion [7]. A similar approach was followed in [24] to provide an ASCLT for sequences of random variables belonging to a fixed Rademacher chaos.

In this paper we prove an ASCLT on the Poisson space, which can be successfully employed to stabilizing (or localized) functionals emerging in stochastic geometry. From the point of view of applications, we provide ASCLTs for the total edge length of the (undirected) $k$-nearest neighbors random graph with $m$ th power weighted edges, the clique count in random geometric graphs, the volume of the set approximation via the Poisson-Voronoi tessellation.

In broad terms, our approach follows the general scheme of [3]. We show a (suitable) abstract ASCLT on the Poisson space. To this aim, we exploit some inequalities for Malliavin's operators on the Poisson space, recently proved in [11], which allow us to express the conditions of the Ibragimov-Lifshits criterion in terms of conditions involving only the first two Malliavin's derivatives of functionals of the Poisson measure. The application to stabilizing functionals relies, instead, on the use of some inequalities on moments of Malliavin's gradients and related probabilities for such functionals, which have recently been proved in [8]. The specific application to the above mentioned geometric quantities is based on estimates for the variance of the corresponding functionals proved in [16, 17, 20, 21] and some arguments in [8] used to check locality of the corresponding functionals.

The paper is organized as follows. In Section 2 we state the main results of the paper, i.e., $(i)$ an ASCLT for localized functionals of the Poisson measure, (ii) ASCLTs for the geometric quantities described above, (iii) an abstract ASCLT on the Poisson space. The proofs of such results are 
given in Sections 3, 4 and 5, respectively.

\section{Main results}

Throughout this paper we consider a probability space $(\Omega, \mathcal{F}, \mathbb{P})$, let $(\mathbb{X}, X)$ be an arbitrary measurable space, let $\mu$ be a $\sigma$-finite measure on $\mathbb{X}$ with $\mu(\mathbb{X})>0$ and denote by $\eta$ the Poisson random measure on $\mathbb{X}$ with intensity measure $\mu$. Formally, we view $\eta$ as a random element on $\mathbf{N}_{\mathbb{X}}$, the space of integer-valued and simple $\sigma$-finite measures $\nu$ equipped with the smallest $\sigma$-field $\mathcal{N}_{\mathbb{X}}$ that makes the mappings $\nu \mapsto \nu(B)$ measurable for any $B \in \mathcal{X}$. When explicitly stated, for ease of notation, we identify a counting measure with its support.

\subsection{An ASCLT for stabilizing functionals on the Poisson space}

Let $\mathbb{Y}$ be a full-dimensional Borel set of $\mathbb{R}^{d}$ and let $\eta$ be the Poisson random measure on $\mathbb{X}:=\mathbb{R}^{d}$ with intensity measure $\mu(\mathrm{d} x):=\ell_{d}(\mathrm{~d} x)$, the Lebesgue measure on $\mathbb{R}^{d}$. Here and in the next subsection, we identify a counting measure $\nu \in \mathbf{N}_{\mathbb{R}^{d}}$ with its support and we consider the statistic

$$
H_{n}:=\sum_{x \in \eta_{n} \cap \mathbb{Y}} \xi_{n}\left(x, \eta_{n} \cap \mathbb{Y}\right), \quad n \geq 1
$$

where $\eta_{n}:=n^{-1 / d} \eta$, i.e., $\eta_{n}$ is the Poisson random measure on $\mathbb{R}^{d}$ with intensity measure $n \ell_{d}(\mathrm{~d} x)$. The so-called score functions $\left\{\xi_{n}\right\}_{n \geq 1}$ are measurable functions from $\mathbb{Y} \times \mathbf{N}_{\mathbb{Y}}$ to $\mathbb{R}$ and represent the local contribution to the global statistic $H_{n}$. In order to introduce the notion of stabilizing functional considered in this paper, we need some definitions.

For $n \geq 1$, a measurable map $R_{n}: \mathbb{Y} \times \mathbf{N}_{\mathbb{Y}} \rightarrow[0, \infty)$ is called a radius of stabilization for the score function $\xi_{n}$ if, for all $(x, \nu) \in \mathbb{Y} \times \mathbf{N}_{\mathbb{Y}}$ and any finite subset $\mathcal{A} \subset \mathbb{Y}$ with at most 7 points,

$$
\xi_{n}\left(x,(\nu \cup\{x\} \cup \mathcal{A}) \cap B\left(x, R_{n}(x, \nu \cup\{x\})\right)\right)=\xi_{n}(x, \nu \cup\{x\} \cup \mathcal{A}) .
$$

Here $B(x, r):=\left\{y \in \mathbb{Y}:\|x-y\|_{d} \leq r\right\}$ denotes the closed ball of radius $r \geq 0$ centered at $x \in \mathbb{Y}$ and $\|\cdot\|_{d}$ is the Euclidean norm on $\mathbb{R}^{d}$. Loosely speaking, the notion of radius of stabilization says that the value of $\xi_{n}$ at $x$ is wholly determined by the points of $\nu$ at distance at most $R_{n}(x, \nu \cup\{x\})$ from $x$. We emphasize that the assumption " $\mathcal{A}$ has at most 7 points" is not required according to the classical definition of radius of stabilization. In this paper we need this extra hypothesis to place ourselves in the same framework of [8]. A similar comment applies to the following definitions of score functions satisfying a moment condition and score functions with exponentially fast decay.

The score functions $\left\{\xi_{n}\right\}_{n \geq 1}$ are called exponentially stabilizing if there exist radii of stabilization $\left\{R_{n}\right\}_{n \geq 1}$ and constants $C_{\text {stab }}, c_{\text {stab }}, \alpha_{\text {stab }} \in \mathbb{R}_{+}:=(0, \infty)$ such that, for $x \in \mathbb{Y}, r \geq 0$ and 
$n \geq 1$

$$
\mathbb{P}\left(R_{n}\left(x,\left(\eta_{n} \cap \mathbb{Y}\right) \cup\{x\}\right) \geq r\right) \leq C_{s t a b} \exp \left(-c_{s t a b} n^{\alpha_{s t a b} / d_{r} r_{s t a b}}\right) .
$$

Let $p^{\prime} \in[0, \infty)$ be given. We say that the score functions $\left\{\xi_{n}\right\}_{n \geq 1}$ satisfy a $\left(4+p^{\prime}\right)$ th moment condition if there is a constant $C_{p^{\prime}} \in \mathbb{R}_{+}$such that for any finite subset $\mathcal{A} \subset \mathbb{Y}$ with at most 7 points,

$$
\sup _{n \geq 1} \sup _{x \in \mathbb{Y}} \mathbb{E}\left[\left|\xi_{n}\left(x,\left(\eta_{n} \cap \mathbb{Y}\right) \cup\{x\} \cup \mathcal{A}\right)\right|^{4+p^{\prime}}\right] \leq C_{p^{\prime}}
$$

Let $K$ be a Borel subset of $\mathbb{Y}$ and put $\mathrm{d}(x, K):=\inf _{y \in K}\|x-y\|_{d}, x \in \mathbb{Y}$. We say that the score functions $\left\{\xi_{n}\right\}_{n \geq 1}$ decay exponentially fast with the distance to $K$ if there are constants $C_{K}, c_{K}, \alpha_{K} \in \mathbb{R}_{+}$such that for any finite subset $\mathcal{A} \subset \mathbb{Y}$ with at most 7 points,

$$
\mathbb{P}\left(\xi_{n}\left(x,\left(\eta_{n} \cap \mathbb{Y}\right) \cup\{x\} \cup \mathcal{A}\right) \neq 0\right) \leq C_{K} \exp \left(-c_{K} n^{\alpha_{K} / d} \mathrm{~d}(x, K)^{\alpha_{K}}\right),
$$

for $x \in \mathbb{Y}$ and $n \geq 1$.

Definition 2.1 We say that the functionals $H_{n}, n \geq 1$, defined by (2.1) are stabilizing if their score functions enjoy the properties (2.3), (2.4) for some $p^{\prime} \in(0,1]$, and (2.5) for some Borel set $K \subseteq \mathbb{Y}$.

Hereon, we consider stabilizing functionals $H_{n}$ and, for a fixed $p^{\prime \prime} \in\left(0, p^{\prime}\right)$, the quantity

$$
I_{K, n}:=n \int_{\mathbb{Y}} \exp \left(-\frac{p^{\prime \prime} c_{p^{\prime}} n^{\alpha / d} \mathrm{~d}(x, K)^{\alpha}}{2^{2 \alpha+3}\left(4+p^{\prime \prime}\right)}\right) \mathrm{d} x, \quad n \geq 1
$$

where

$$
c_{p^{\prime}}:=p^{\prime}\left(c_{s t a b} \wedge c_{K}\right), \quad \alpha:=\alpha_{s t a b} \wedge \alpha_{K}
$$

and we denote by $a \wedge b$ the minimum between $a, b \in \mathbb{R}$. Throughout this paper, we use the standard Landau notation, i.e., given two sequences $\left\{a_{n}\right\}_{n \geq 1},\left\{b_{n}\right\}_{n \geq 1} \subset \mathbb{R}_{+}$, we write $a_{n}=O\left(b_{n}\right)$ if $\limsup _{n \rightarrow \infty} \frac{a_{n}}{b_{n}}<\infty$.

The following theorem holds.

Theorem 2.2 If the functionals $H_{n}, n \geq 1$, defined by (2.1) are stabilizing, $n^{\tau}=O\left(\mathbb{V a r}\left(H_{n}\right)\right)$ and $I_{K, n}=O\left(n^{\tau}\right)$, for some $\tau \in(0,2)$, then $\left\{F_{n}\right\}_{n \geq 1}, F_{n}:=\left(H_{n}-\mathbb{E}\left[H_{n}\right]\right) / \sqrt{\mathbb{V a r}\left(H_{n}\right)}$, satisfies the ASCLT.

We remark that in the statement of Theorem 2.2 the quantities $\operatorname{Var}\left(H_{n}\right)$ and $I_{K, n}$ are finite and strictly positive for any $n \geq 1$ (this is an implicit consequence of the Landau notation, as defined above, which is employed to compare sequences in $\mathbb{R}_{+}$). We also emphasize that, although 
Theorem 2.2 holds for any scaling regime $\tau \in(0,2)$, all the examples considered in this paper fall either in the class of the volume order scaling (i.e., $\tau=1$ ) or in the class of the surface area order scaling (i.e. $\tau=1-1 / d$ ).

\section{$2.2 \quad$ ASCLTs for stochastic geometry models}

Theorem 2.2 finds a natural application to models emerging in stochastic geometry, such as the total edge length of the $k$-nearest neighbors random graph, the clique count in random geometric graphs and the volume of the set approximation via the Poisson-Voronoi tessellation.

\subsubsection{An ASCLT for the total edge length of the $k$-nearest neighbors random graph}

Let $\mathbb{Y}$ be a full-dimensional Borel set of $\mathbb{R}^{d}$ and $n, k \geq 1$ two integers. For $x \in \eta_{n} \cap \mathbb{Y}$, we denote by $V_{k}\left(x, \eta_{n} \cap \mathbb{Y}\right)$ the set of the $k$ nearest neighbors of $x$, i.e., the $k$ closest points of $x$ in $\left(\eta_{n} \cap \mathbb{Y}\right) \backslash\{x\}$. The (undirected) $k$-nearest neighbors random graph $N G_{k}\left(\eta_{n} \cap \mathbb{Y}\right)$ is the random graph with vertex set $\eta_{n} \cap \mathbb{Y}$ obtained by including an edge $\{x, y\}$ if $y \in V_{k}\left(x, \eta_{n} \cap \mathbb{Y}\right)$ and/or $x \in V_{k}\left(y, \eta_{n} \cap \mathbb{Y}\right)$. For all $m \geq 0$, we set

$$
\xi^{(m)}\left(x, \eta_{n} \cap \mathbb{Y}\right):=\sum_{y \in V_{k}\left(x, \eta_{n} \cap \mathbb{Y}\right)} \rho^{(m)}(x, y)
$$

where

$$
\rho^{(m)}(x, y):=\mathbf{1}\left\{x \in V_{k}\left(y, \eta_{n} \cap \mathbb{Y}\right)\right\} \frac{\|x-y\|_{d}^{m}}{2}+\mathbf{1}\left\{x \notin V_{k}\left(y, \eta_{n} \cap \mathbb{Y}\right)\right\}\|x-y\|_{d}^{m} .
$$

The total edge length of the (undirected) $k$-nearest neighbors random graph on $\eta_{n} \cap \mathbb{Y}$ with $m$ th power weighted edges is

$$
L_{N G_{k}}^{(m)}\left(\eta_{n} \cap \mathbb{Y}\right):=\sum_{x \in \eta_{n} \cap \mathbb{Y}} \xi^{(m)}\left(x, \eta_{n} \cap \mathbb{Y}\right) .
$$

Note that $L_{N G_{k}}^{(0)}\left(\eta_{n} \cap \mathbb{Y}\right)$ is the number of edges and $L_{N G_{k}}^{(1)}\left(\eta_{n} \cap \mathbb{Y}\right)$ is the total edge length. When $\mathbb{Y}$ is a full-dimensional compact and convex subset of $\mathbb{R}^{d}$, the central limit theorem and a quantitative central limit theorem (in the Kolmogorov distance) for the sequence $\left\{L_{N G_{k}}^{(m)}\left(\eta_{n} \cap \mathbb{Y}\right)\right\}_{n \geq 1}$ are proved in [16] and [1], respectively. Here, we provide an ASCLT for such sequence.

Set

$$
F_{n}:=\frac{L_{N G_{k}}^{(m)}\left(\eta_{n} \cap \mathbb{Y}\right)-\mathbb{E}\left[L_{N G_{k}}^{(m)}\left(\eta_{n} \cap \mathbb{Y}\right)\right]}{\sqrt{\operatorname{Var}\left(L_{N G_{k}}^{(m)}\left(\eta_{n} \cap \mathbb{Y}\right)\right)}}, \quad n \geq 1 .
$$

The following corollary holds.

Corollary 2.3 If $\mathbb{Y}$ is a full-dimensional compact and convex subset of $\mathbb{R}^{d}$, then $\left\{F_{n}\right\}_{n \geq 1}$ defined by (2.8) satisfies the ASCLT. 


\subsubsection{An ASCLT for the clique count in random geometric graphs}

Let $\mathbb{Y}$ be a full-dimensional subset of $\mathbb{R}^{d}$ and consider the random geometric graph $G\left(\eta_{n} \cap \mathbb{Y}, r\right)$, $n \geq 1, r \in \mathbb{R}_{+}$, where two nodes $x, y \in \eta_{n} \cap \mathbb{Y}$ are joined with an edge if $\|x-y\|_{d} \leq r$. We recall that $k+1$ nodes of $G\left(\eta_{n} \cap \mathbb{Y}, r\right)$ form a clique of order $k+1$ if each pair of them is connected by an edge. The number of cliques of order $k+1$ in $G\left(\eta_{n} \cap \mathbb{Y}, r\right)$, denoted by $\mathcal{C}_{k}\left(\eta_{n} \cap \mathbb{Y}, r\right)$, is a central statistic in topological data analysis. A quantitative central limit theorem (in the Kolmogorov distance) for the sequence $\left\{\mathcal{C}_{k}\left(\eta_{n} \cap \mathbb{Y}, r n^{-1 / d}\right)\right\}_{n \geq 1}$ has been proved in [14, Chapter 3. Here, we give an ASCLT for such sequence.

Set

$$
F_{n}:=\frac{\mathcal{C}_{k}\left(\eta_{n} \cap \mathbb{Y}, r n^{-1 / d}\right)-\mathbb{E}\left[\mathcal{C}_{k}\left(\eta_{n} \cap \mathbb{Y}, r n^{-1 / d}\right)\right]}{\sqrt{\operatorname{Var}\left(\mathcal{C}_{k}\left(\eta_{n} \cap \mathbb{Y}, r n^{-1 / d}\right)\right)}}, \quad n \geq 1 .
$$

The following corollary holds.

Corollary 2.4 If $\mathbb{Y}$ is a full-dimensional subset of $\mathbb{R}^{d}$ such that $\ell_{d}(\mathbb{Y})<\infty$, then $\left\{F_{n}\right\}_{n \geq 1}$ defined by (2.9) satisfies the ASCLT.

\subsubsection{An ASCLT for the volume of the set approximation via the Poisson-Voronoi tessellation}

Let $\mathbb{Y}:=[-1 / 2,1 / 2]^{d}, d \geq 2$, and let $A \subset(-1 / 2,1 / 2)^{d}$ be a full-dimensional subset of $\mathbb{R}^{d}$. Assume that we observe $\eta_{n} \cap \mathbb{Y}$ and that the only information about $A$ at our disposal is which points of $\eta_{n} \cap \mathbb{Y}$ lie in $A$, i.e., we have the partition of the process $\eta_{n} \cap \mathbb{Y}$ into $\eta_{n} \cap A$ and $\left(\eta_{n} \cap \mathbb{Y}\right) \backslash A$. To reconstruct the set $A$ just by the information at our disposal, we approximate $A$ by its PoissonVoronoi approximation $A_{n}$, i.e., the set of all points in $\mathbb{Y}$ which are closer to $\eta_{n} \cap A$ than to $\left(\eta_{n} \cap \mathbb{Y}\right) \backslash A$. Formally,

$$
A_{n}:=\bigcup_{x \in \eta_{n} \cap A} \mathrm{C}\left(x, \eta_{n} \cap \mathbb{Y}\right)
$$

where $\mathrm{C}\left(x, \eta_{n} \cap \mathbb{Y}\right)$ is the Poisson-Voronoi cell generated by $\eta_{n} \cap \mathbb{Y}$ with nucleus $x \in \eta_{n} \cap \mathbb{Y}$, i.e.,

$$
\mathrm{C}\left(x, \eta_{n} \cap \mathbb{Y}\right):=\left\{y \in \mathbb{Y}:\|y-x\|_{d} \leq\|y-z\|_{d} \forall z \in \eta_{n} \cap \mathbb{Y}\right\}
$$

Note that $\mathrm{C}\left(x, \eta_{n} \cap \mathbb{Y}\right)$ is a random convex polytope and that the Poisson-Voronoi tessellation (or

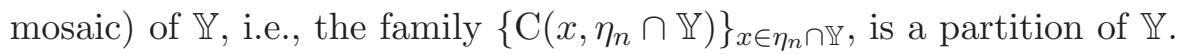

If $A$ is compact and convex it follows, respectively from [15] and [18, that, as $n \rightarrow \infty$, $\ell_{d}\left(A_{n}\right) \rightarrow \ell_{d}(A)$ almost surely, and $\mathbb{E}\left[\ell_{d}\left(A_{n}\right)\right] \rightarrow \ell_{d}(A)$. A quantitative central limit theorem (in the Kolmogorov distance) for the sequence $\left\{\ell_{d}\left(A_{n}\right)\right\}_{n \geq 1}$ is proved in $[8$. Here, we give an ASCLT for such sequence. 
Put

$$
F_{n}:=\frac{\ell_{d}\left(A_{n}\right)-\mathbb{E}\left[\ell_{d}\left(A_{n}\right)\right]}{\sqrt{\operatorname{Var}\left(\ell_{d}\left(A_{n}\right)\right)}}, \quad n \geq 1 .
$$

The following corollary holds.

Corollary 2.5 If $A$ is compact and convex then $\left\{F_{n}\right\}_{n \geq 1}$ defined by (2.10) satisfies the ASCLT.

\subsection{An ASCLT on the Poisson space}

As already mentioned in the Introduction, in this section we present an abstract ASCLT for the Poisson space, which provides theoretical foundation for the proof of Theorem 2.2, To state such general result we need to introduce some additional notation.

By $L_{\eta}^{r}, r \in \mathbb{R}_{+}$, we denote the space of all random variables $F \in L^{r}(\mathbb{P})$ such that $F=f(\eta)$ $\mathbb{P}$-a.s. for some measurable function $f: \mathbf{N}_{\mathbb{X}} \rightarrow \mathbb{R}$, where $L^{r}(\mathbb{P})$ is the set of random variables $X: \Omega \rightarrow \mathbb{R}$ such that $\mathbb{E}\left[|X|^{r}\right]<\infty$. For $F \in L_{\eta}^{2}, F=f(\eta)$, and $x_{1}, x_{2} \in \mathbb{X}$ we define the Malliavin derivative operators

$$
\begin{gathered}
D_{x_{1}} F:=f\left(\eta+\varepsilon_{x_{1}}\right)-f(\eta), \\
D_{x_{1}, x_{2}}^{2} F:=D_{x_{2}}\left(D_{x_{1}} F\right)=f\left(\eta+\varepsilon_{x_{1}}+\varepsilon_{x_{2}}\right)-f\left(\eta+\varepsilon_{x_{2}}\right)-f\left(\eta+\varepsilon_{x_{1}}\right)+f(\eta) .
\end{gathered}
$$

For $H_{i} \in L_{\eta}^{2}$ with $\operatorname{Var}\left(H_{i}\right)>0, i=1,2,3,4$, and positive constants $p, q \in \mathbb{R}_{+}$(defined in the statement of Theorem (2.6), we put

$$
\begin{gathered}
\boldsymbol{\Gamma}\left(H_{1}, H_{2}, H_{3}, H_{4}\right)^{2} \\
:=\boldsymbol{\Lambda}\left(H_{1}, H_{2}, H_{3}, H_{4}\right)^{2}+\frac{1}{\operatorname{Var}\left(H_{1}\right) \operatorname{Var}\left(H_{2}\right)} \int_{\mathbb{X}} \psi_{x}\left(H_{1}, q /(2(4+q))\right) \psi_{x}\left(H_{2}, q /(2(4+q))\right) \mu(\mathrm{d} x), \\
\psi_{x}(G, \beta):=\int_{\mathbb{X}} \mathbb{P}\left(D_{x_{1}, x}^{2} G \neq 0\right)^{\beta} \mu\left(\mathrm{d} x_{1}\right), \quad x \in \mathbb{X}, G \in L_{\eta}^{2}, \beta \in \mathbb{R}_{+}, \\
\boldsymbol{\Lambda}\left(H_{1}, H_{2}, H_{3}, H_{4}\right)^{2}:=\frac{1}{\prod_{i=1}^{4} \mathbb{V a r}\left(H_{i}\right)^{1 / 2}} \int_{\mathbb{X}} \psi_{x}\left(H_{1}, q /(4(4+q))\right) \psi_{x}\left(H_{2}, q /(4(4+q))\right) \mu(\mathrm{d} x), \\
\boldsymbol{\Theta}\left(H_{1}, H_{2}\right):=\frac{1}{\prod_{i=1}^{2} \operatorname{Var}\left(H_{i}\right)^{1 / 2}} \int_{\mathbb{X}} \mathbb{P}\left(D_{x} H_{1} \neq 0\right)^{p /(4(4+p))} \mathbb{P}\left(D_{x} H_{2} \neq 0\right)^{p /(4(4+p))} \mu(\mathrm{d} x), \\
\boldsymbol{\Gamma}_{2}\left(H_{1}\right):=\frac{1}{\boldsymbol{V}_{1}\left(H_{1}\right)^{2}:=\boldsymbol{\Gamma}\left(H_{1}, H_{1}, H_{1}, H_{1}\right)^{2},} \int_{\mathbb{X}} \mathbb{P}\left(D_{x} H_{1} \neq 0\right)^{(1+p) /(4+p)} \mu(\mathrm{d} x) .
\end{gathered}
$$

The following theorem holds, where we denote by $a \vee b$ the maximum between $a, b \in \mathbb{R}$. 
Theorem 2.6 Assume $\left\{H_{n}\right\}_{n \geq 1} \subset L_{\eta}^{2}, \operatorname{Var}\left(H_{n}\right)>0, n \geq 1$, and that there exist constants $c, p, q \in \mathbb{R}_{+}$such that

$$
\begin{gathered}
\sup _{n \geq 1} \mathbb{E}\left[\left|D_{x_{1}} H_{n}\right|^{4+p}\right] \leq c, \quad \mu \text {-a.e. } x_{1} \in \mathbb{X} \\
\left.\sup _{n \geq 1} \mathbb{E}\left[\left|D_{x_{1}, x_{2}}^{2} H_{n}\right|^{4+q}\right]\right\} \leq c, \quad \mu^{\otimes 2} \text {-a.e. }\left(x_{1}, x_{2}\right) \in \mathbb{X}^{2} .
\end{gathered}
$$

Moreover, suppose

$$
\begin{gathered}
\lim _{n \rightarrow \infty} \boldsymbol{\Gamma}_{1}\left(H_{n}\right)=\lim _{n \rightarrow \infty} \boldsymbol{\Gamma}_{2}\left(H_{n}\right)=0, \\
\sum_{n \geq 2} \frac{1}{n(\log n)^{2}} \sum_{k=1}^{n} \frac{\boldsymbol{\Gamma}_{i}\left(H_{k}\right)}{k}<\infty, \quad i=1,2 \\
\sum_{n \geq 2} \frac{1}{n(\log n)^{3}} \sum_{l=1}^{n} \sum_{k=1}^{l} \frac{\boldsymbol{\Theta}\left(H_{k}, H_{l}\right)}{k l}<\infty, \\
\sum_{n \geq 2} \frac{1}{n(\log n)^{3}} \sum_{l=1}^{n} \sum_{k=1}^{l} \frac{\boldsymbol{\Gamma}\left(H_{k}, H_{l}, H_{k}, H_{l}\right)}{k l}<\infty, \\
\sum_{n \geq 2} \frac{1}{n(\log n)^{3}} \sum_{l=1}^{n} \sum_{k=1}^{l} \frac{\boldsymbol{\Lambda}_{\max }\left(H_{k}, H_{l}\right)}{k l}<\infty,
\end{gathered}
$$

where

$$
\begin{aligned}
\boldsymbol{\Lambda}_{\max }\left(H_{k}, H_{l}\right):= & \boldsymbol{\Lambda}\left(H_{k}, H_{k}, H_{k}, H_{l}\right) \vee \boldsymbol{\Lambda}\left(H_{l}, H_{l}, H_{l}, H_{k}\right) \vee \boldsymbol{\Lambda}\left(H_{k}, H_{k}, H_{l}, H_{l}\right) \vee \boldsymbol{\Lambda}\left(H_{l}, H_{l}, H_{k}, H_{k}\right) \\
& \vee \boldsymbol{\Lambda}\left(H_{l}, H_{k}, H_{k}, H_{k}\right) \vee \boldsymbol{\Lambda}\left(H_{k}, H_{l}, H_{l}, H_{l}\right) \vee \boldsymbol{\Lambda}\left(H_{k}, H_{l}, H_{l}, H_{k}\right) .
\end{aligned}
$$

Then $\left\{F_{n}\right\}_{n \geq 1}, F_{n}:=\left(H_{n}-\mathbb{E}\left[H_{n}\right]\right) / \sqrt{\mathbb{V a r}\left(H_{n}\right)}$, satisfies the ASCLT.

\section{Proof of Theorem 2.2}

The proof of Theorem 2.2 exploits some recent inequalities, stated in Subsection 3.1, concerning moments and probabilities of Malliavin's gradients of localized functionals due to Lachiéze-Rey, Schulte and Yukich [8]. The proof of the theorem is then given in Subsection 3.2.

We start introducing some notation. Throughout we assume that the functionals $H_{n}, n \geq 1$, defined by (2.1) are stabilizing. Recalling that here we identify a counting measure $\nu \in \mathbf{N}_{\mathbb{R}^{d}}$ with its support, for $y_{1}, y_{2} \in \mathbb{Y}$, we set

$$
\mathcal{D}_{y_{1}} H_{n}:=\sum_{x \in\left(\eta_{n} \cap \mathbb{Y}\right) \cup\left\{y_{1}\right\}} \xi_{n}\left(x,\left(\eta_{n} \cap \mathbb{Y}\right) \cup\left\{y_{1}\right\}\right)-\sum_{x \in \eta_{n} \cap \mathbb{Y}} \xi_{n}\left(x, \eta_{n} \cap \mathbb{Y}\right)
$$

and

$$
\mathcal{D}_{y_{1}, y_{2}}^{2} H_{n}:=\mathcal{D}_{y_{2}}\left(\mathcal{D}_{y_{1}} H_{n}\right)=\mathcal{D}_{y_{1}, y_{2}}^{2,+} H_{n}-\mathcal{D}_{y_{1}} H_{n}
$$


where

$$
\mathcal{D}_{y_{1}, y_{2}}^{2,+} H_{n}:=\sum_{x \in\left(\eta_{n} \cap \mathbb{Y}\right) \cup\left\{y_{1}, y_{2}\right\}} \xi_{n}\left(x,\left(\eta_{n} \cap \mathbb{Y}\right) \cup\left\{y_{1}, y_{2}\right\}\right)-\sum_{x \in\left(\eta_{n} \cap \mathbb{Y}\right) \cup\left\{y_{2}\right\}} \xi_{n}\left(x,\left(\eta_{n} \cap \mathbb{Y}\right) \cup\left\{y_{2}\right\}\right) .
$$

We remark that these quantities are well-defined thanks to Lemma 3.1 of the next subsection.

\subsection{Preliminary lemmas}

The following lemma is an immediate consequence of Lemma 5.5 in 8 ].

Lemma 3.1 If the functionals $H_{n}, n \geq 1$, defined by (2.1) are stabilizing, then, for any fixed $p^{\prime \prime} \in\left(0, p^{\prime}\right)$, there exists a constant $c\left(p^{\prime \prime}\right) \in \mathbb{R}_{+}$(only depending on the constants $C_{\text {stab }}, c_{\text {stab }}, \alpha_{\text {stab }}$, $p^{\prime}$ and $C_{p^{\prime}}$ appearing in the definition of stabilizing functional) such that

$$
\sup _{n \geq 1} \sup _{y \in \mathbb{Y}} \mathbb{E}\left[\left|\mathcal{D}_{y} H_{n}\right|^{4+p^{\prime \prime}}\right] \leq c\left(p^{\prime \prime}\right) \quad \text { and } \sup _{n \geq 1} \sup _{y_{1}, y_{2} \in \mathbb{Y}} \mathbb{E}\left[\left|\mathcal{D}_{y_{1}, y_{2}}^{2,+} H_{n}\right|^{4+p^{\prime \prime}}\right] \leq c\left(p^{\prime \prime}\right)
$$

The following lemma is an immediate consequence of Lemma 5.10 in [8] (see the inequalities (5.6) and (5.8) therein).

Lemma 3.2 If the functionals $H_{n}, n \geq 1$, defined by (2.1) are stabilizing, then, for any fixed $\beta \in \mathbb{R}_{+}$there exists a constant $\tilde{C}_{\beta} \in \mathbb{R}_{+}$(not depending on $n$ ) such that

$$
n \int_{\mathbb{Y}}\left(n \int_{\mathbb{Y}} \mathbb{P}\left(\mathcal{D}_{y_{1}, y_{2}}^{2} H_{n} \neq 0\right)^{\beta} \mathrm{d} y_{1}\right)^{2} \mathrm{~d} y_{2} \leq \tilde{C}_{\beta} n \int_{\mathbb{Y}} \exp \left(-\frac{c_{p^{\prime}} \beta}{2^{2 \alpha+1}}\left(n^{1 / d} \mathrm{~d}(x, K)\right)^{\alpha}\right) \mathrm{d} x
$$

and

$$
n \int_{\mathbb{Y}} \mathbb{P}\left(\mathcal{D}_{y} H_{n} \neq 0\right)^{\beta} \mathrm{d} y \leq \tilde{C}_{\beta} n \int_{\mathbb{Y}} \exp \left(-\frac{c_{p^{\prime}} \beta}{2^{\alpha+1}}\left(n^{1 / d} \mathrm{~d}(x, K)\right)^{\alpha}\right) \mathrm{d} x,
$$

where the constants $c_{p^{\prime}}$ and $\alpha$ are defined in (2.7).

We conclude this subsection with a lemma which relates the operators $D$ and $D^{2}$ with the operators $\mathcal{D}$ and $\mathcal{D}^{2}$, respectively.

Lemma 3.3 If the functionals $H_{n}, n \geq 1$, defined by (2.1) are stabilizing and $I_{K, n}<\infty$, then $H_{n} \in L^{2}(\mathbb{P})$ and, for any $x_{1}, x_{2} \in \mathbb{R}^{d}$,

$$
D_{x_{1}} H_{n}=\mathbf{1}\left\{n^{-1 / d} x_{1} \in \mathbb{Y}\right\} \mathcal{D}_{n^{-1 / d} x_{1}} H_{n}
$$

and

$$
D_{x_{1}, x_{2}}^{2} H_{n}=\mathbf{1}\left\{n^{-1 / d} x_{1}, n^{-1 / d} x_{2} \in \mathbb{Y}\right\} \mathcal{D}_{n^{-1 / d} x_{1}, n^{-1 / d} x_{2}}^{2} H_{n}
$$


Proof. We divide the proof in two steps. In the first step we check the square integrability of $H_{n}$, in the second step we verify the relations among the operators.

Step 1: Checking $H_{n} \in L^{2}(\mathbb{P}), n \geq 1$. Throughout this proof $p^{\prime \prime} \in\left(0, p^{\prime}\right)$ is the constant appearing in the definition of $I_{K, n}$. We shall check later on that, for any $0<\gamma \leq 2^{2 \alpha+3}\left(4+p^{\prime \prime}\right)$ and $x \in \mathbb{Y}$,

$$
\exp \left(-c_{K} n^{\alpha_{K} / d} \mathrm{~d}(x, K)^{\alpha_{K}} / \gamma\right) \leq \hat{C} \exp \left(-p^{\prime \prime} c_{p^{\prime}} n^{\alpha / d} \mathrm{~d}(x, K)^{\alpha} /\left(2^{2 \alpha+3}\left(4+p^{\prime \prime}\right)\right)\right),
$$

for a suitable constant $\hat{C} \in \mathbb{R}_{+}$not depending on $n$, where the constants $c_{p^{\prime}}$ and $\alpha$ are defined in (2.7). Setting $M:=\sum_{x \in \eta_{n} \cap \mathbb{Y}} \mathbf{1}\left(\xi_{n}\left(x, \eta_{n} \cap \mathbb{Y}\right) \neq 0\right)$, by Jensen's inequality we have

$$
\begin{aligned}
H_{n}^{2}= & M^{2}\left(\sum_{x \in \eta_{n} \cap \mathbb{Y}} \frac{\mathbf{1}\left\{\xi_{n}\left(x, \eta_{n} \cap \mathbb{Y}\right) \neq 0\right\}}{M} \xi_{n}\left(x, \eta_{n} \cap \mathbb{Y}\right)\right)^{2} \\
\leq & M \sum_{x \in \eta_{n} \cap \mathbb{Y}} \mathbf{1}\left\{\xi_{n}\left(x, \eta_{n} \cap \mathbb{Y}\right) \neq 0\right\} \xi_{n}\left(x, \eta_{n} \cap \mathbb{Y}\right)^{2} \\
= & \sum_{x, x^{\prime} \in \eta_{n} \cap \mathbb{Y}} \mathbf{1}\left\{\xi_{n}\left(x^{\prime}, \eta_{n} \cap \mathbb{Y}\right) \neq 0, \xi_{n}\left(x, \eta_{n} \cap \mathbb{Y}\right) \neq 0\right\} \xi_{n}\left(x, \eta_{n} \cap \mathbb{Y}\right)^{2} \\
= & \sum_{x \in \eta_{n} \cap \mathbb{Y}} \mathbf{1}\left\{\xi_{n}\left(x, \eta_{n} \cap \mathbb{Y}\right) \neq 0\right\} \xi_{n}\left(x, \eta_{n} \cap \mathbb{Y}\right)^{2} \\
& \quad+\sum_{x, x^{\prime} \in \eta_{n} \cap \mathbb{Y}: x \neq x^{\prime}} \mathbf{1}\left\{\xi_{n}\left(x^{\prime}, \eta_{n} \cap \mathbb{Y}\right) \neq 0, \xi_{n}\left(x, \eta_{n} \cap \mathbb{Y}\right) \neq 0\right\} \xi_{n}\left(x, \eta_{n} \cap \mathbb{Y}\right)^{2} .
\end{aligned}
$$

By the (multivariate) Mecke formula (see e.g. [10], Chapter 4), the Cauchy-Schwarz inequality, Hölder's inequality, (2.4), (2.5) and (3.3), we get

$$
\begin{aligned}
& \mathbb{E}\left[H_{n}^{2}\right] \leq n \int_{\mathbb{Y}} \mathbb{E}\left[\mathbf{1}\left\{\xi_{n}\left(x,\left(\eta_{n} \cap \mathbb{Y}\right) \cup\{x\}\right) \neq 0\right\} \xi_{n}\left(x,\left(\eta_{n} \cap \mathbb{Y}\right) \cup\{x\}\right)^{2}\right] \mathrm{d} x \\
& +n^{2} \int_{\mathbb{Y}^{2}} \mathbb{E}\left[\mathbf{1}\left\{\xi_{n}\left(x^{\prime},\left(\eta_{n} \cap \mathbb{Y}\right) \cup\left\{x, x^{\prime}\right\}\right) \neq 0, \xi_{n}\left(x,\left(\eta_{n} \cap \mathbb{Y}\right) \cup\left\{x, x^{\prime}\right\}\right) \neq 0\right\}\right. \\
& \left.\quad \times \xi_{n}\left(x,\left(\eta_{n} \cap \mathbb{Y}\right) \cup\left\{x, x^{\prime}\right\}\right)^{2}\right] \mathrm{d} x \mathrm{~d} x^{\prime} \\
& \leq n \int_{\mathbb{Y}} \mathbb{P}\left(\xi_{n}\left(x,\left(\eta_{n} \cap \mathbb{Y}\right) \cup\{x\}\right) \neq 0\right)^{1 / 2} \mathbb{E}\left[\xi_{n}\left(x,\left(\eta_{n} \cap \mathbb{Y}\right) \cup\{x\}\right)^{4}\right]^{1 / 2} \mathrm{~d} x \\
& \quad+n^{2} \int_{\mathbb{Y}^{2}} \mathbb{E}\left[\mathbf{1}\left\{\xi_{n}\left(x^{\prime},\left(\eta_{n} \cap \mathbb{Y}\right) \cup\left\{x, x^{\prime}\right\}\right) \neq 0, \xi_{n}\left(x,\left(\eta_{n} \cap \mathbb{Y}\right) \cup\left\{x, x^{\prime}\right\}\right) \neq 0\right\}\right]^{1 / 2} \\
& \quad \times \mathbb{E}\left[\xi_{n}\left(x,\left(\eta_{n} \cap \mathbb{Y}\right) \cup\left\{x, x^{\prime}\right\}\right)^{4}\right]^{1 / 2} \mathrm{~d} x \mathrm{~d} x^{\prime} \\
& \leq n \int_{\mathbb{Y}} \mathbb{P}\left(\xi_{n}\left(x,\left(\eta_{n} \cap \mathbb{Y}\right) \cup\{x\}\right) \neq 0\right)^{1 / 2} \mathbb{E}\left[\xi_{n}\left(x,\left(\eta_{n} \cap \mathbb{Y}\right) \cup\{x\}\right)^{4}\right]^{1 / 2} \mathrm{~d} x \\
& \quad+n^{2} \int_{\mathbb{Y}^{2}} \mathbb{P}\left(\xi_{n}\left(x^{\prime},\left(\eta_{n} \cap \mathbb{Y}\right) \cup\left\{x, x^{\prime}\right\}\right) \neq 0\right)^{1 / 4} \mathbb{P}\left(\xi_{n}\left(x,\left(\eta_{n} \cap \mathbb{Y}\right) \cup\left\{x, x^{\prime}\right\}\right) \neq 0\right)^{1 / 4} \\
& \quad \times \mathbb{E}\left[\xi_{n}\left(x,\left(\eta_{n} \cap \mathbb{Y}\right) \cup\left\{x, x^{\prime}\right\}\right)^{4}\right]^{1 / 2} \mathrm{~d} x \mathrm{~d} x^{\prime} \\
& \quad n \int_{\mathbb{Y}} \mathbb{P}\left(\xi_{n}\left(x,\left(\eta_{n} \cap \mathbb{Y}\right) \cup\{x\}\right) \neq 0\right)^{1 / 2} \mathbb{E}\left[\xi_{n}\left(x,\left(\eta_{n} \cap \mathbb{Y}\right) \cup\{x\}\right)^{4+p^{\prime}}\right]^{2 /\left(4+p^{\prime}\right)} \mathrm{d} x \\
& \quad+n^{2} \int_{\mathbb{Y}^{2}} \mathbb{P}\left(\xi_{n}\left(x^{\prime},\left(\eta_{n} \cap \mathbb{Y}\right) \cup\left\{x, x^{\prime}\right\}\right) \neq 0\right)^{1 / 4} \mathbb{P}\left(\xi_{n}\left(x,\left(\eta_{n} \cap \mathbb{Y}\right) \cup\left\{x, x^{\prime}\right\}\right) \neq 0\right)^{1 / 4}
\end{aligned}
$$




$$
\begin{aligned}
& \times \mathbb{E}\left[\xi_{n}\left(x,\left(\eta_{n} \cap \mathbb{Y}\right) \cup\left\{x, x^{\prime}\right\}\right)^{4+p^{\prime}}\right]^{2 /\left(4+p^{\prime}\right)} \mathrm{d} x \mathrm{~d} x^{\prime} \\
& \leq C_{p^{\prime}}^{2 /\left(4+p^{\prime}\right)} n \int_{\mathbb{Y}} \mathbb{P}\left(\xi_{n}\left(x,\left(\eta_{n} \cap \mathbb{Y}\right) \cup\{x\}\right) \neq 0\right)^{1 / 2} \mathrm{~d} x \\
& \quad+C_{p^{\prime}}^{2 /\left(4+p^{\prime}\right)} n^{2} \int_{\mathbb{Y}^{2}} \mathbb{P}\left(\xi_{n}\left(x^{\prime},\left(\eta_{n} \cap \mathbb{Y}\right) \cup\left\{x, x^{\prime}\right\}\right) \neq 0\right)^{1 / 4} \mathbb{P}\left(\xi_{n}\left(x,\left(\eta_{n} \cap \mathbb{Y}\right) \cup\left\{x, x^{\prime}\right\}\right) \neq 0\right)^{1 / 4} \mathrm{~d} x \mathrm{~d} x^{\prime} \\
& \leq C_{K}^{1 / 2} C_{p^{\prime}}^{2 /\left(4+p^{\prime}\right)}\left[n \int_{\mathbb{Y}} \exp \left(-c_{K} n^{\alpha_{K} / d} \mathrm{~d}(x, K)^{\alpha_{K}} / 2\right) \mathrm{d} x+\left(n \int_{\mathbb{Y}} \exp \left(-c_{K} n^{\alpha_{K} / d} \mathrm{~d}(x, K)^{\alpha_{K}} / 4\right) \mathrm{d} x\right)^{2}\right] \\
& \leq C^{\prime}\left[I_{K, n}+\left(I_{K, n}\right)^{2}\right]<\infty,
\end{aligned}
$$

for a suitable constant $C^{\prime} \in \mathbb{R}_{+}$. It remains to check (3.3). Since $\gamma \leq 2^{2 \alpha+3}\left(4+p^{\prime \prime}\right)$, we have

$$
\begin{aligned}
& \exp \left(-c_{K} n^{\alpha_{K} / d} \mathrm{~d}(x, K)^{\alpha_{K}} / \gamma\right) \\
& \leq \exp \left(-\frac{p^{\prime \prime} c_{p^{\prime}}}{2^{2 \alpha+3}\left(4+p^{\prime \prime}\right)}\left(n^{1 / d} \mathrm{~d}(x, K)\right)^{\alpha_{K}}\right) \\
&= \mathbf{1}\left\{n^{1 / d} \mathrm{~d}(x, K) \leq 1\right\} \exp \left(-\frac{p^{\prime \prime} c_{p^{\prime}}}{2^{2 \alpha+3}\left(4+p^{\prime \prime}\right)}\left(n^{1 / d} \mathrm{~d}(x, K)\right)^{\alpha_{K}}\right) \\
&+\mathbf{1}\left\{n^{1 / d} \mathrm{~d}(x, K)>1\right\} \exp \left(-\frac{p^{\prime \prime} c_{p^{\prime}}}{2^{2 \alpha+3}\left(4+p^{\prime \prime}\right)}\left(n^{1 / d} \mathrm{~d}(x, K)\right)^{\alpha_{K}}\right) \\
& \leq \mathbf{1}\left\{n^{1 / d} \mathrm{~d}(x, K) \leq 1\right\} \exp \left(-\frac{p^{\prime \prime} c_{p^{\prime}}}{2^{2 \alpha+3}\left(4+p^{\prime \prime}\right)}\left(n^{1 / d} \mathrm{~d}(x, K)\right)^{\alpha}\right) \\
& \quad \times \exp \left(\frac{p^{\prime \prime} c_{p^{\prime}}}{2^{2 \alpha+3}\left(4+p^{\prime \prime}\right)}\left[\left(n^{1 / d} \mathrm{~d}(x, K)\right)^{\alpha}-\left(n^{1 / d} \mathrm{~d}(x, K)\right)^{\alpha_{K}}\right]\right) \\
& \quad \exp \left(-\frac{p^{\prime \prime} c_{p^{\prime}}}{2^{2 \alpha+3}\left(4+p^{\prime \prime}\right)}\left(n^{1 / d} \mathrm{~d}(x, K)\right)^{\alpha}\right) \\
& \leq\left(\mathbf{1}\left\{n^{1 / d} \mathrm{~d}(x, K) \leq 1\right\} \exp \left(\frac{p^{\prime \prime} c_{p^{\prime}}}{2^{2 \alpha+3}\left(4+p^{\prime \prime}\right)}\right)+1\right) \exp \left(-\frac{p^{\prime \prime} c_{p^{\prime}}}{2^{2 \alpha+3}\left(4+p^{\prime \prime}\right)}\left(n^{1 / d} \mathrm{~d}(x, K)\right)^{\alpha}\right) \\
& \leq \hat{C} \exp \left(-\frac{p^{\prime \prime} c_{p^{\prime}}}{2^{2 \alpha+3}\left(4+p^{\prime \prime}\right)}\left(n^{1 / d} \mathrm{~d}(x, K)\right)^{\alpha}\right),
\end{aligned}
$$

where we used that, for $a>1$, the function $x \mapsto a^{x}$ is non-decreasing on $[0, \infty)$.

Step 2: Checking the relations among the operators. For any $x_{1} \in \mathbb{R}^{d}$, we have

$$
\begin{aligned}
& D_{x_{1}} H_{n}= \mathbf{1}\left\{n^{-1 / d} x_{1} \in \mathbb{Y}\right\}\left(\xi_{n}\left(n^{-1 / d} x_{1},\left(\eta_{n} \cup\left\{n^{-1 / d} x_{1}\right\}\right) \cap \mathbb{Y}\right)\right. \\
&\left.+\sum_{z \in \eta_{n} \cap \mathbb{Y}} \xi_{n}\left(z,\left(\eta_{n} \cup\left\{n^{-1 / d} x_{1}\right\}\right) \cap \mathbb{Y}\right)-\sum_{z \in \eta_{n} \cap \mathbb{Y}} \xi_{n}\left(z, \eta_{n} \cap \mathbb{Y}\right)\right) \\
&=\mathbf{1}\left\{n^{-1 / d} x_{1} \in \mathbb{Y}\right\} \mathcal{D}_{n^{-1 / d} x_{1}} H_{n} .
\end{aligned}
$$

The relation (3.2) can be verified by a similar computation. 


\subsection{Proof of Theorem 2.2}

We apply Theorem 2.6 and start noticing that the square integrability of the functionals $H_{n}, n \geq 1$, follows by Lemma 3.3. In the next steps we check all the other conditions of Theorem 2.6. Hereafter, $p^{\prime \prime} \in\left(0, p^{\prime}\right)$ is the constant involved in the definition of $I_{K, n}$.

Step 1: Checking Conditions (2.11) and (2.12). By (3.1) and Lemma 3.1, we have

$$
\sup _{n \geq 1} \sup _{x \in \mathbb{R}^{d}} \mathbb{E}\left[\left|D_{x} H_{n}\right|^{4+p^{\prime \prime}}\right] \leq c\left(p^{\prime \prime}\right) .
$$

By (3.2), the inequality $|a+b|^{r} \leq 2^{r-1}\left(|a|^{r}+|b|^{r}\right), r \geq 1$, and again Lemma 3.1, we have

$$
\begin{aligned}
\sup _{n \geq 1} \sup _{x_{1}, x_{2} \in \mathbb{R}^{d}} \mathbb{E}\left[\left|D_{x_{1}, x_{2}}^{2} H_{n}\right|^{4+p^{\prime \prime}}\right] & \leq \sup _{n \geq 1} \sup _{y_{1}, y_{2} \in \mathbb{Y}} \mathbb{E}\left[\left|\mathcal{D}_{y_{1}, y_{2}}^{2} H_{n}\right|^{4+p^{\prime \prime}}\right] \\
& \leq 2^{3+p^{\prime \prime}} \sup _{n \geq 1} \sup _{y_{1}, y_{2} \in \mathbb{Y}}\left(\mathbb{E}\left[\left|\mathcal{D}_{y_{1}, y_{2}}^{2,+} H_{n}\right|^{4+p^{\prime \prime}}\right]+\mathbb{E}\left[\left|\mathcal{D}_{y_{1}} H_{n}\right|^{4+p^{\prime \prime}}\right]\right) \\
& \leq 2^{4+p^{\prime \prime}} c\left(p^{\prime \prime}\right) .
\end{aligned}
$$

So conditions (2.11) and (2.12) are verified with $p=q=p^{\prime \prime}$ and $c=2^{4+p^{\prime \prime}} c\left(p^{\prime \prime}\right)$. To conclude the proof it remains to check (2.13), (2.14), (2.15), (2.16) and (2.17) where the quantities $\boldsymbol{\Lambda}, \boldsymbol{\Gamma}, \boldsymbol{\Theta}$ and $\boldsymbol{\Gamma}_{2}$ involve $p^{\prime \prime}$ in place of $p$ and $q$. This task is accomplished in the Steps 2-6.

Step 2: Two Preliminary Inequalities. Let $\beta \in \mathbb{R}_{+}$be fixed. By (3.2) and Lemma 3.2, we have

$$
\begin{aligned}
\int_{\mathbb{R}^{d}} \psi_{x}\left(H_{n}, \beta\right)^{2} \mathrm{~d} x=\int_{\mathbb{R}^{d}}\left(\int_{\mathbb{R}^{d}} \mathbb{P}\left(D_{x_{1}, x}^{2} H_{n} \neq 0\right)^{\beta} \mathrm{d} x_{1}\right)^{2} \mathrm{~d} x \\
=\int_{\mathbb{R}^{d}}\left(\int_{\mathbb{R}^{d}} \mathbb{P}\left(\mathbf{1}\left\{n^{-1 / d} x_{1}, n^{-1 / d} x \in \mathbb{Y}\right\} \mathcal{D}_{n^{-1 / d} x_{1}, n^{-1 / d} x}^{2} H_{n} \neq 0\right)^{\beta} \mathrm{d} x_{1}\right)^{2} \mathrm{~d} x \\
=n \int_{\mathbb{Y}}\left(n \int_{\mathbb{Y}} \mathbb{P}\left(\mathcal{D}_{x_{1}, x}^{2} H_{n} \neq 0\right)^{\beta} \mathrm{d} x_{1}\right)^{2} \mathrm{~d} x \\
\leq \tilde{C}_{\beta} n \int_{\mathbb{Y}} \exp \left(-\frac{c_{p^{\prime}} \beta}{2^{2 \alpha+1}}\left(n^{1 / d} \mathrm{~d}(x, K)\right)^{\alpha}\right) \mathrm{d} x
\end{aligned}
$$

and

$$
\begin{aligned}
\int_{\mathbb{R}^{d}} \mathbb{P}\left(D_{x} H_{n} \neq 0\right)^{\beta} \mathrm{d} x & =\int_{\mathbb{R}^{d}} \mathbf{1}\left\{n^{-1 / d} x \in \mathbb{Y}\right\} \mathbb{P}\left(\mathcal{D}_{n^{-1 / d} x} H_{n} \neq 0\right)^{\beta} \mathrm{d} x \\
& =n \int_{\mathbb{Y}} \mathbb{P}\left(\mathcal{D}_{y} H_{n} \neq 0\right)^{\beta} \mathrm{d} y \\
& \leq \tilde{C}_{\beta} n \int_{\mathbb{Y}} \exp \left(-\frac{c_{p^{\prime}} \beta}{2^{\alpha+1}}\left(n^{1 / d} \mathrm{~d}(x, K)\right)^{\alpha}\right) \mathrm{d} x \\
& \leq \tilde{C}_{\beta} n \int_{\mathbb{Y}} \exp \left(-\frac{c_{p^{\prime}} \beta}{2^{2 \alpha+1}}\left(n^{1 / d} \mathrm{~d}(x, K)\right)^{\alpha}\right) \mathrm{d} x .
\end{aligned}
$$


Since $\psi_{x}(G, \beta)$ is a non-increasing function of $\beta$, from relation (3.4) we get

$$
\begin{aligned}
& \int_{\mathbb{R}^{d}} \psi_{x}\left(H_{n}, p^{\prime \prime} /\left(4\left(4+p^{\prime \prime}\right)\right)\right)^{2} \mathrm{~d} x \bigvee \int_{\mathbb{R}^{d}} \psi_{x}\left(H_{n}, p^{\prime \prime} /\left(2\left(4+p^{\prime \prime}\right)\right)\right)^{2} \mathrm{~d} x \\
& =\int_{\mathbb{R}^{d}} \psi_{x}\left(H_{n}, p^{\prime \prime} /\left(4\left(4+p^{\prime \prime}\right)\right)\right)^{2} \mathrm{~d} x \leq \tilde{C}_{p^{\prime \prime} /\left(4\left(4+p^{\prime \prime}\right)\right)} I_{K, n} .
\end{aligned}
$$

Similarly, from (3.5), since $\mathbb{P}\left(D_{x} H_{n} \neq 0\right)^{\beta}$ is non-increasing in $\beta$ we obtain

$$
\begin{aligned}
\int_{\mathbb{R}^{d}} \mathbb{P}\left(D_{x} H_{n} \neq 0\right)^{\left(1+p^{\prime \prime}\right) /\left(4+p^{\prime \prime}\right)} \mathrm{d} x & \leq \int_{\mathbb{R}^{d}} \mathbb{P}\left(D_{x} H_{n} \neq 0\right)^{p^{\prime \prime} /\left(2\left(4+p^{\prime \prime}\right)\right)} \mathrm{d} x \\
& \leq \int_{\mathbb{R}^{d}} \mathbb{P}\left(D_{x} H_{n} \neq 0\right)^{p^{\prime \prime} /\left(4\left(4+p^{\prime \prime}\right)\right)} \mathrm{d} x \leq \tilde{C}_{p^{\prime \prime} /\left(4\left(4+p^{\prime \prime}\right)\right)} I_{K, n} .
\end{aligned}
$$

Step 3: Checking Conditions (2.13) and (2.14). From now on in this proof, $C>0$ denotes a generic positive constant (not depending on $n$ ) which may vary from line to line. Note that by the assumptions it follows

$$
\operatorname{Var}\left(H_{n}\right) \geq C n^{\tau}, \quad \text { for all } n \text { large enough }
$$

and

$$
I_{K, n} \leq C n^{\tau}, \quad \text { for all } n \text { large enough. }
$$

By (3.8), (3.6) and (3.9), we have

$$
\begin{aligned}
\boldsymbol{\Gamma}_{1}\left(H_{n}\right) & =\frac{1}{\operatorname{Var}\left(H_{n}\right)} \sqrt{\int_{\mathbb{R}^{d}} \psi_{x}\left(H_{n}, p^{\prime \prime} /\left(4\left(4+p^{\prime \prime}\right)\right)\right)^{2} \mathrm{~d} x+\int_{\mathbb{R}^{d}} \psi_{x}\left(H_{n}, p^{\prime \prime} /\left(2\left(4+p^{\prime \prime}\right)\right)\right)^{2} \mathrm{~d} x} \\
& \leq \frac{C}{n^{\tau}} \sqrt{\int_{\mathbb{R}^{d}} \psi_{x}\left(H_{n}, p^{\prime \prime} /\left(4\left(4+p^{\prime \prime}\right)\right)\right)^{2} \mathrm{~d} x+\int_{\mathbb{R}^{d}} \psi_{x}\left(H_{n}, p^{\prime \prime} /\left(2\left(4+p^{\prime \prime}\right)\right)\right)^{2} \mathrm{~d} x} \\
& \leq C n^{-\tau / 2}, \quad \text { for all } n \text { large enough. }
\end{aligned}
$$

Similarly, by (3.8), (3.7) and (3.9), we have

$$
\begin{aligned}
\boldsymbol{\Gamma}_{2}\left(H_{n}\right) & =\frac{1}{\operatorname{Var}\left(H_{n}\right)^{3 / 2}} \int_{\mathbb{R}^{d}} \mathbb{P}\left(D_{x} H_{n} \neq 0\right)^{\left(1+p^{\prime \prime}\right) /\left(4+p^{\prime \prime}\right)} \mathrm{d} x \\
& \leq \frac{C}{n^{3 \tau / 2}} \int_{\mathbb{R}^{d}} \mathbb{P}\left(D_{x} H_{n} \neq 0\right)^{\left(1+p^{\prime \prime}\right) /\left(4+p^{\prime \prime}\right)} \mathrm{d} x \\
& \leq C n^{-\tau / 2}, \quad \text { for all } n \text { large enough. }
\end{aligned}
$$

Relation (2.13) follows immediately by (3.11) and (3.13). By (3.11) and (3.13) we also have, for some $\bar{n} \geq 2$ large enough,

$$
\sum_{n \geq \bar{n}} \frac{1}{n(\log n)^{2}} \sum_{k=\bar{n}}^{n} \frac{\Gamma_{i}\left(H_{k}\right)}{k} \leq C \sum_{n \geq \bar{n}} \frac{1}{n(\log n)^{2}} \sum_{k \geq \bar{n}} \frac{1}{k^{1+\tau / 2}}<\infty, \quad i=1,2 .
$$


Therefore (2.14) follows since $\boldsymbol{\Gamma}_{i}\left(H_{n}\right)<\infty$, for any $i=1,2$ and $n \geq 1$ as a consequence of the following assumptions: $\operatorname{Var}\left(H_{n}\right) \in \mathbb{R}_{+}$and $I_{K, n} \in \mathbb{R}_{+}$for $n \geq 1$, (3.10), (3.12), (3.6) and (3.7) (which hold for any $n \geq 1$ ).

Step 4: Checking Condition (2.15). By (3.8), (3.7) and (3.9), we have

$$
\begin{aligned}
\Theta\left(H_{k}, H_{l}\right) & =\frac{1}{\sqrt{\operatorname{Var}\left(H_{k}\right) \mathbb{V a r}\left(H_{l}\right)}} \int_{\mathbb{R}^{d}} \mathbb{P}\left(D_{x} H_{k} \neq 0\right)^{p^{\prime \prime} /\left(4\left(4+p^{\prime \prime}\right)\right)} \mathbb{P}\left(D_{x} H_{l} \neq 0\right)^{p^{\prime \prime} /\left(4\left(4+p^{\prime \prime}\right)\right)} \mathrm{d} x \\
& \leq \frac{1}{\sqrt{\operatorname{Var}\left(H_{k}\right) \mathbb{V a r}\left(H_{l}\right)}} \int_{\mathbb{R}^{d}} \mathbb{P}\left(D_{x} H_{k} \neq 0\right)^{p^{\prime \prime} /\left(4\left(4+p^{\prime \prime}\right)\right)} \mathrm{d} x \\
& \leq \frac{C}{\sqrt{k^{\tau}} \sqrt{l^{\tau}}} \int_{\mathbb{R}^{d}} \mathbb{P}\left(D_{x} H_{k} \neq 0\right)^{p^{\prime \prime} /\left(4\left(4+p^{\prime \prime}\right)\right)} \mathrm{d} x \\
& \leq C\left(\frac{k}{l}\right)^{\tau / 2}, \quad \text { for all } k, l \text { large enough. }
\end{aligned}
$$

Therefore, for some $\bar{k} \geq 2$ large enough,

$$
\begin{aligned}
\sum_{n \geq \bar{k}} \frac{1}{n(\log n)^{3}} \sum_{l=\bar{k}}^{n} \sum_{k=\bar{k}}^{l} \frac{\Theta\left(H_{k}, H_{l}\right)}{k l} & \leq C \sum_{n \geq \bar{k}} \frac{1}{n(\log n)^{3}} \sum_{l=\bar{k}}^{n} \frac{1}{l^{1+\tau / 2}} \sum_{k=\bar{k}}^{l} k^{\tau / 2-1} \\
& \leq C \sum_{n \geq \bar{k}} \frac{1}{n(\log n)^{3}} \sum_{l=\bar{k}}^{n} \frac{1}{l^{1+\tau / 2}} \sum_{k=2}^{l} \int_{k-1}^{k} k^{\tau / 2-1} \mathrm{~d} x \\
& \leq C \sum_{n \geq \bar{k}} \frac{1}{n(\log n)^{3}} \sum_{l=\bar{k}}^{n} \frac{1}{l^{1+\tau / 2}} \int_{1}^{l} x^{\tau / 2-1} \mathrm{~d} x \\
& =C \sum_{n \geq \bar{k}} \frac{1}{n(\log n)^{3}} \sum_{l=\bar{k}}^{n} \frac{1}{l^{1+\tau / 2}}\left(l^{\tau / 2}-1\right) \\
& \leq C \sum_{n \geq \bar{k}} \frac{1}{n(\log n)^{3}} \sum_{l=\bar{k}}^{n} \frac{1}{l} \\
& \leq C \sum_{n \geq \bar{k}} \frac{1}{n(\log n)^{2}}<\infty
\end{aligned}
$$

where in (3.16) we used that $0<\tau<2$. By (3.8), the Cauchy-Schwarz inequality, (3.7) and (3.9), for $\bar{k} \geq 2$ large enough and $n \geq \bar{k}$,

$$
\begin{aligned}
& \sum_{l=\bar{k}}^{n} \sum_{k=1}^{\bar{k}-1} \frac{\boldsymbol{\Theta}\left(H_{k}, H_{l}\right)}{k l} \\
& \quad \leq C \sum_{l=\bar{k}}^{n} \sum_{k=1}^{\bar{k}-1} \frac{1}{k \mathbb{V} a r\left(H_{k}\right)^{1 / 2} l^{1+\tau / 2}}\left(\int_{\mathbb{R}^{d}} \mathbb{P}\left(D_{x} H_{k} \neq 0\right)^{p^{\prime \prime} /\left(2\left(4+p^{\prime \prime}\right)\right)} \mathrm{d} x\right)^{1 / 2}\left(\int_{\mathbb{R}^{d}} \mathbb{P}\left(D_{x} H_{l} \neq 0\right)^{p^{\prime \prime} /\left(2\left(4+p^{\prime \prime}\right)\right)} \mathrm{d} x\right)^{1 / 2} \\
& \quad \leq C \sum_{k=1}^{\bar{k}-1} \frac{\sqrt{I_{K, k}}}{k \mathbb{V a r}\left(H_{k}\right)^{1 / 2}} \sum_{l=\bar{k}}^{n} \frac{1}{l} \\
& \quad \leq C \log n
\end{aligned}
$$


Relations (3.17) and (3.18) yield, for $\bar{k} \geq 2$ large enough,

$$
\sum_{n \geq \bar{k}} \frac{1}{n(\log n)^{3}} \sum_{l=\bar{k}}^{n} \sum_{k=1}^{l} \frac{\Theta\left(H_{k}, H_{l}\right)}{k l}<\infty
$$

This relation implies (2.15) since $\boldsymbol{\Theta}\left(H_{k}, H_{l}\right)<\infty$ for any $k, l \geq 1$ as a consequence of the following assumptions: $\operatorname{Var}\left(H_{n}\right) \in \mathbb{R}_{+}$and $I_{K, n} \in \mathbb{R}_{+}$for any $n \geq 1$, (3.14) and (3.7) (which hold for any $k, l \geq 1)$.

Step 5: Checking Condition (2.16). For $k, l, m, n \geq 1$, using that $\psi_{x}(G, \beta)$ is non-increasing in $\beta$ and the Cauchy-Schwarz inequality, we have

$$
\begin{aligned}
& \boldsymbol{\Gamma}\left(H_{k}, H_{l}, H_{m}, H_{n}\right)^{2} \\
& \leq \frac{1}{\sqrt{\operatorname{Var}\left(H_{k}\right) \operatorname{Var}\left(H_{l}\right) \operatorname{Var}\left(H_{m}\right) \operatorname{Var}\left(H_{n}\right)}} \int_{\mathbb{R}^{d}} \psi_{x}\left(H_{k}, p^{\prime \prime} /\left(4\left(4+p^{\prime \prime}\right)\right)\right) \psi_{x}\left(H_{l}, p^{\prime \prime} /\left(4\left(4+p^{\prime \prime}\right)\right)\right) \mathrm{d} x \\
& +\frac{1}{\operatorname{Var}\left(H_{k}\right) \mathbb{V a r}\left(H_{l}\right)} \int_{\mathbb{R}^{d}} \psi_{x}\left(H_{k}, p^{\prime \prime} /\left(2\left(4+p^{\prime \prime}\right)\right)\right) \psi_{x}\left(H_{l}, p^{\prime \prime} /\left(2\left(4+p^{\prime \prime}\right)\right)\right) \mathrm{d} x \\
& \leq\left(\frac{1}{\sqrt{\operatorname{Var}\left(H_{k}\right) \operatorname{Var}\left(H_{l}\right) \mathbb{V a r}\left(H_{m}\right) \operatorname{Var}\left(H_{n}\right)}}+\frac{1}{\operatorname{Var}\left(H_{k}\right) \operatorname{Var}\left(H_{l}\right)}\right) \\
& \times \int_{\mathbb{R}^{d}} \psi_{x}\left(H_{k}, p^{\prime \prime} /\left(4\left(4+p^{\prime \prime}\right)\right)\right) \psi_{x}\left(H_{l}, p^{\prime \prime} /\left(4\left(4+p^{\prime \prime}\right)\right)\right) \mathrm{d} x \\
& \leq\left(\frac{1}{\sqrt{\operatorname{Var}\left(H_{k}\right) \operatorname{Var}\left(H_{l}\right) \operatorname{Var}\left(H_{m}\right) \operatorname{Var}\left(H_{n}\right)}}+\frac{1}{\operatorname{Var}\left(H_{k}\right) \operatorname{Var}\left(H_{l}\right)}\right) \\
& \times\left(\int_{\mathbb{R}^{d}} \psi_{x}\left(H_{k}, p^{\prime \prime} /\left(4\left(4+p^{\prime \prime}\right)\right)\right)^{2} \mathrm{~d} x\right)^{1 / 2}\left(\int_{\mathbb{R}^{d}} \psi_{x}\left(H_{l}, p^{\prime \prime} /\left(4\left(4+p^{\prime \prime}\right)\right)\right)^{2} \mathrm{~d} x\right)^{1 / 2} .
\end{aligned}
$$

By this relation, (3.8), (3.6) and (3.9), for $l, k$ large enough

$$
\boldsymbol{\Gamma}\left(H_{k}, H_{l}, H_{k}, H_{l}\right) \leq \frac{C}{(k l)^{\tau / 4}} .
$$

Therefore, for some $\bar{k} \geq 2$ large enough

$$
\sum_{n \geq \bar{k}} \frac{1}{n(\log n)^{3}} \sum_{l=\bar{k}}^{n} \sum_{k=\bar{k}}^{l} \frac{\boldsymbol{\Gamma}\left(H_{k}, H_{l}, H_{k}, H_{l}\right)}{k l} \leq C \sum_{n \geq \bar{k}} \frac{1}{n(\log n)^{3}} \sum_{l=\bar{k}}^{n} \frac{1}{l^{1+\tau / 4}} \sum_{k=\bar{k}}^{l} \frac{1}{k^{1+\tau / 4}}<\infty .
$$

For $\bar{k} \geq 2$ large enough, by (3.19), (3.6), (3.9) and (3.8), we have

$$
\begin{aligned}
& \sum_{n \geq \bar{k}} \frac{1}{n(\log n)^{3}} \sum_{l=\bar{k}}^{n} \sum_{k=1}^{\bar{k}-1} \frac{\boldsymbol{\Gamma}\left(H_{k}, H_{l}, H_{k}, H_{l}\right)}{k l} \\
& \leq C \sum_{n \geq \bar{k}} \frac{1}{n(\log n)^{3}} \sum_{l=\bar{k}}^{n} \sum_{k=1}^{\bar{k}-1} \frac{1}{k l} \sqrt{\frac{I_{K, k}^{1 / 2} I_{K, l}^{1 / 2}}{\operatorname{Var}\left(H_{k}\right) \operatorname{Var}\left(H_{l}\right)}}
\end{aligned}
$$




$$
\begin{aligned}
& \leq C\left(\sum_{k=1}^{\bar{k}-1} \frac{1}{k} \sqrt{\frac{I_{K, k}^{1 / 2}}{\operatorname{Var}\left(H_{k}\right)}}\right) \sum_{n \geq \bar{k}} \frac{1}{n(\log n)^{3}}\left(\sum_{l=\bar{k}}^{n} \frac{1}{l} \sqrt{\frac{I_{K, l}^{1 / 2}}{\operatorname{Var}\left(H_{l}\right)}}\right) \\
& \leq C \sum_{n \geq \bar{k}} \frac{1}{n(\log n)^{3}} \sum_{l=\bar{k}}^{n} \frac{1}{l} \sqrt{\frac{I_{K, l}^{1 / 2}}{\operatorname{Var}\left(H_{l}\right)}} \\
& \leq C \sum_{n \geq \bar{k}} \frac{1}{n(\log n)^{3}} \sum_{l=\bar{k}}^{n} \frac{1}{l^{1+\tau / 4}}<\infty .
\end{aligned}
$$

This relation, along with (3.20), yields (2.16) since $\boldsymbol{\Gamma}\left(H_{k}, H_{l}, H_{k}, H_{l}\right)<\infty$ for any $k, l \geq 1$ as a consequence of the following assumptions: $\operatorname{Var}\left(H_{n}\right) \in \mathbb{R}_{+}$and $I_{K, n} \in \mathbb{R}_{+}$for any $n \geq 1$, (3.19) and (3.6) (which hold for any $k, l \geq 1$ ).

Step 6: Checking Condition (2.17). By the trivial inequality

$$
\mathbf{\Lambda}\left(H_{k}, H_{l}, H_{m}, H_{n}\right) \leq \boldsymbol{\Gamma}\left(H_{k}, H_{l}, H_{m}, H_{n}\right),
$$

(3.19), the inequality $(a+b)^{1 / 2} \leq a^{1 / 2}+b^{1 / 2}, a, b \geq 0$, and (3.6), for $k, l \geq 1$, we have

$$
\begin{aligned}
& \boldsymbol{\Lambda}_{\text {max }}\left(H_{k}, H_{l}\right) \leq C\left(\frac{1}{\operatorname{Var}\left(H_{k}\right)^{3 / 4} \operatorname{Var}\left(H_{l}\right)^{1 / 4}}+\frac{1}{\operatorname{Var}\left(H_{k}\right)}\right) I_{K, k}^{1 / 2} \\
& \vee\left(\frac{1}{\operatorname{Var}\left(H_{l}\right)^{3 / 4} \operatorname{Var}\left(H_{k}\right)^{1 / 4}}+\frac{1}{\operatorname{Var}\left(H_{l}\right)}\right) I_{K, l}^{1 / 2} \\
& \vee\left(\frac{1}{\left(\operatorname{Var}\left(H_{k}\right) \operatorname{Var}\left(H_{l}\right)\right)^{1 / 2}}+\frac{1}{\operatorname{Var}\left(H_{k}\right)}\right) I_{K, k}^{1 / 2} \\
& \vee\left(\frac{1}{\left(\operatorname{Var}\left(H_{k}\right) \mathbb{V} a r\left(H_{l}\right)\right)^{1 / 2}}+\frac{1}{\operatorname{Var}\left(H_{l}\right)}\right) I_{K, l}^{1 / 2} \\
& \vee\left(\frac{1}{\operatorname{Var}\left(H_{k}\right)^{3 / 4} \operatorname{Var}\left(H_{l}\right)^{1 / 4}}+\frac{1}{\left(\operatorname{Var}\left(H_{k}\right) \operatorname{Var}\left(H_{l}\right)\right)^{1 / 2}}\right)\left(I_{K, k} I_{K, l}\right)^{1 / 4} \\
& \vee\left(\frac{1}{\operatorname{Var}\left(H_{l}\right)^{3 / 4} \operatorname{Var}\left(H_{k}\right)^{1 / 4}}+\frac{1}{\left(\operatorname{Var}\left(H_{k}\right) \operatorname{Var}\left(H_{l}\right)\right)^{1 / 2}}\right)\left(I_{K, k} I_{K, l}\right)^{1 / 4} \\
& \vee \frac{\left(I_{K, k} I_{K, l}\right)^{1 / 4}}{\left(\operatorname{Var}\left(H_{k}\right) \mathbb{V a r}\left(H_{l}\right)\right)^{1 / 2}} \\
& =C\left(\frac{I_{K, k}^{1 / 2}}{\operatorname{Var}\left(H_{k}\right)^{3 / 4}} \frac{1}{\operatorname{Var}\left(H_{l}\right)^{1 / 4}}+\frac{I_{K, k}^{1 / 2}}{\operatorname{Var}\left(H_{k}\right)}\right) \\
& \vee\left(\frac{1}{\operatorname{Var}\left(H_{k}\right)^{1 / 4}} \frac{I_{K, l}^{1 / 2}}{\operatorname{Var}\left(H_{l}\right)^{3 / 4}}+\frac{I_{K, l}^{1 / 2}}{\operatorname{Var}\left(H_{l}\right)}\right) \\
& \vee\left(\frac{I_{K, k}^{1 / 2}}{\left(\operatorname{Var}\left(H_{k}\right)\right)^{1 / 2}} \frac{1}{\left(\operatorname{Var}\left(H_{l}\right)\right)^{1 / 2}}+\frac{I_{K, k}^{1 / 2}}{\operatorname{Var}\left(H_{k}\right)}\right) \\
& \vee\left(\frac{1}{\left(\operatorname{Var}\left(H_{k}\right)\right)^{1 / 2}} \frac{I_{K, l}^{1 / 2}}{\left(\mathbb{V a r}\left(H_{l}\right)\right)^{1 / 2}}+\frac{I_{K, l}^{1 / 2}}{\operatorname{Var}\left(H_{l}\right)}\right)
\end{aligned}
$$




$$
\begin{aligned}
& \vee\left(\frac{\left(I_{K, k} I_{K, l}\right)^{1 / 4}}{\operatorname{Var}\left(H_{k}\right)^{3 / 4} \operatorname{Var}\left(H_{l}\right)^{1 / 4}}+\frac{\left(I_{K, k} I_{K, l}\right)^{1 / 4}}{\left(\mathbb{V a r}\left(H_{k}\right) \operatorname{Var}\left(H_{l}\right)\right)^{1 / 2}}\right) \\
& \vee\left(\frac{\left(I_{K, k} I_{K, l}\right)^{1 / 4}}{\operatorname{Var}\left(H_{l}\right)^{3 / 4} \operatorname{Var}\left(H_{k}\right)^{1 / 4}}+\frac{\left(I_{K, k} I_{K, l}\right)^{1 / 4}}{\left(\mathbb{V a r}\left(H_{k}\right) \operatorname{Var}\left(H_{l}\right)\right)^{1 / 2}}\right) \\
& \vee \frac{\left(I_{K, k} I_{K, l}\right)^{1 / 4}}{\left(\operatorname{Var}\left(H_{k}\right) \operatorname{Var}\left(H_{l}\right)\right)^{1 / 2}} .
\end{aligned}
$$

Consequently, by (3.9) and (3.8), for some $\bar{k} \geq 2$ large enough and $l \geq k \geq \bar{k}$, we have

$$
\boldsymbol{\Lambda}_{\max }\left(H_{k}, H_{l}\right) \leq \frac{C}{k^{\tau / 2}} .
$$

Therefore, for some $\bar{k} \geq 2$ large enough, we have

$$
\sum_{n \geq \bar{k}} \frac{1}{n(\log n)^{3}} \sum_{l=\bar{k}}^{n} \sum_{k=\bar{k}}^{l} \frac{\boldsymbol{\Lambda}_{\max }\left(H_{k}, H_{l}\right)}{k l} \leq C \sum_{n \geq \bar{k}} \frac{1}{n(\log n)^{3}} \sum_{l=\bar{k}}^{n} \frac{1}{l} \sum_{k=\bar{k}}^{l} \frac{1}{k^{1+\tau / 2}}<\infty .
$$

Let $\bar{k} \geq 2$ large enough and $l \geq \bar{k}>k$, let $C_{i}(k) \in \mathbb{R}_{+}, i=0,1, \ldots, 12$, denote suitable constants, depending on $k$ but not on $l$, which may vary from line to line. By (3.21), (3.9) and (3.8), we have

$$
\begin{aligned}
\boldsymbol{\Lambda}_{\text {max }}\left(H_{k}, H_{l}\right) & \leq\left(C_{1}(k) \frac{1}{\operatorname{Var}\left(H_{l}\right)^{1 / 4}}+C_{2}(k)\right) \\
& \vee\left(C_{3}(k) \frac{I_{K, l}^{1 / 2}}{\operatorname{Var}\left(H_{l}\right)^{3 / 4}}+C_{4}(k) \frac{1}{\operatorname{Var}\left(H_{l}\right)}\right) \vee\left(C_{5}(k) \frac{1}{\left(\mathbb{V a r}\left(H_{l}\right)\right)^{1 / 2}}+C_{6}(k)\right) \\
& \vee\left(C_{7}(k) \frac{I_{K, l}^{1 / 2}}{\left(\mathbb{V a r}\left(H_{l}\right)\right)^{1 / 2}}+\frac{I_{K, l}^{1 / 2}}{\operatorname{Var}\left(H_{l}\right)}\right) \vee\left(C_{8}(k) \frac{\left(I_{K, l}\right)^{1 / 4}}{\operatorname{Var}\left(H_{l}\right)^{1 / 4}}+C_{9}(k) \frac{\left(I_{K, l}\right)^{1 / 4}}{\left(\mathbb{V a r}\left(H_{l}\right)\right)^{1 / 2}}\right) \\
& \vee\left(C_{10}(k) \frac{\left(I_{K, l}\right)^{1 / 4}}{\operatorname{Var}\left(H_{l}\right)^{3 / 4}}+C_{11}(k) \frac{\left(I_{K, l}\right)^{1 / 4}}{\left(\mathbb{V a r}\left(H_{l}\right)\right)^{1 / 2}}\right) \vee C_{12}(k) \frac{\left(I_{K, l}\right)^{1 / 4}}{\left(\mathbb{V a r}\left(H_{l}\right)\right)^{1 / 2}} \\
& \leq\left(C_{1}(k) l^{-\tau / 4}+C_{2}(k)\right) \vee\left(C_{3}(k) l^{-\tau / 4}+C_{4}(k) l^{-\tau / 2}\right) \\
& \vee\left(C_{5}(k) l^{-\tau / 2}+C_{6}(k)\right) \vee\left(C_{7}(k)+l^{-\tau / 2}\right) \\
& \vee\left(C_{8}(k)+C_{9}(k) l^{-\tau / 4}\right) \vee\left(C_{10}(k) l^{-\tau / 2}+C_{11}(k) l^{-\tau / 4}\right) \vee C_{12}(k) l^{-\tau / 4} \\
& \leq C_{0}(k) .
\end{aligned}
$$

Therefore, for $\bar{k} \geq 2$ large enough, we have

$$
\sum_{n \geq \bar{k}} \frac{1}{n(\log n)^{3}} \sum_{l=\bar{k}}^{n} \sum_{k=1}^{\bar{k}-1} \frac{\Lambda_{\max }\left(H_{k}, H_{l}\right)}{k l} \leq C_{0}(k) \sum_{n \geq \bar{k}} \frac{1}{n(\log n)^{3}} \sum_{l=\bar{k}}^{n} \frac{1}{l}<\infty .
$$

This relation, along with (3.22), yields (2.17) since $\boldsymbol{\Lambda}_{\max }\left(H_{k}, H_{l}\right)<\infty$ for any $k, l \geq 1$ as a consequence of the following assumptions: $\operatorname{Var}\left(H_{n}\right) \in \mathbb{R}_{+}$and $I_{K, n} \in \mathbb{R}_{+}$for any $n \geq 1$, and (3.21) (which holds for any $k, l \geq 1$ ). 


\section{Proofs of Corollaries 2.3, 2.4 and 2.5}

\subsection{Proof of Corollary 2.3}

Set $H_{n}:=n^{m / d} L_{N G_{k}}^{(m)}\left(\eta_{n} \cap \mathbb{Y}\right)$ and note that, for $F_{n}$ defined in (2.8), we have

$$
F_{n}=\frac{H_{n}-\mathbb{E}\left[H_{n}\right]}{\sqrt{\operatorname{Var}\left(H_{n}\right)}}
$$

The claim follows by Theorem 2.2 with $\tau=1$. Indeed: ( $i$ ) By Theorem 2.1 and Lemma 6.3 in [16] (see also the discussion on the related variance bounds in [8]) we have $n^{1-2 m / d}=O\left(\operatorname{Var}\left(L_{N G_{k}}^{(m)}\left(\eta_{n} \cap\right.\right.\right.$ $\mathbb{Y})$ ), and so $n=O\left(\operatorname{Var}\left(H_{n}\right)\right)$. (ii) One can show that the functionals $H_{n}, n \geq 1$, are stabilizing following the proof of Theorem 3.1 in [8]. We briefly sketch the line of the proof. For $n \geq 1$ and $x \in \eta_{n} \cap \mathbb{Y}$, we set $\xi_{n}\left(x, \eta_{n} \cap \mathbb{Y}\right):=n^{m / d} \xi^{(m)}\left(x, \eta_{n} \cap \mathbb{Y}\right)$. It turns out that $H_{n}=\sum_{x \in \eta_{n} \cap \mathbb{Y}} \xi_{n}\left(x, \eta_{n} \cap \mathbb{Y}\right)$ and that the score functions $\xi_{n}, n \geq 1$, satisfy (2.3), (2.4) and (2.5) with $K:=\mathbb{Y}$. (iii) By (2.6) easily follows $I_{K, n}=I_{\mathbb{Y}, n}=n \ell_{d}(\mathbb{Y})$.

\subsection{Proof of Corollary 2.4}

Setting $H_{n}:=\mathcal{C}_{k}\left(\eta_{n} \cap \mathbb{Y}, r n^{-1 / d}\right), n \geq 1$, the claim follows by Theorem 2.2 with $\tau=1$. Indeed: $(i)$ As noticed in the proof of Theorem 2.5 in Section 7 of [17] (see also Remark(iv) on p. 967 in [8]), we have $\inf _{n \geq 1} \operatorname{Var}\left(H_{n}\right) / n>0$, and so $n=O\left(\operatorname{Var}\left(H_{n}\right)\right)$. (ii) One can show that the functionals $H_{n}, n \geq 1$, are stabilizing following the proof of Theorem 3.15 in [8]. We briefly sketch the line of the proof. For $n \geq 1$ and $x \in \eta_{n} \cap \mathbb{Y}$, we denote by $\xi_{n}\left(x, \eta_{n} \cap \mathbb{Y}\right)$ the number of cliques of order $k+1$ in $G\left(\eta_{n} \cap \mathbb{Y}, r n^{-1 / d}\right)$ containing $x$. It turns out that $H_{n}=\sum_{x \in \eta_{n} \cap \mathbb{Y}} \xi_{n}\left(x, \eta_{n} \cap \mathbb{Y}\right)$ and that the score functions $\xi_{n}, n \geq 1$, satisfy (2.3), (2.4) and (2.5) with $K:=\mathbb{Y}$. (iii) By (2.6) easily follows $I_{K, n}=I_{\mathbb{Y}, n}=n \ell_{d}(\mathbb{Y})$.

\subsection{Proof of Corollary 2.5}

Set $H_{n}:=n\left(\ell_{d}\left(A_{n}\right)-\ell_{d}(A)\right), n \geq 1$, and note that, for $F_{n}$ defined in (2.10), we have

$$
F_{n}=\frac{H_{n}-\mathbb{E}\left[H_{n}\right]}{\sqrt{\operatorname{Var}\left(H_{n}\right)}} .
$$

The claim follows by applying Theorem 2.2 with $\tau:=1-1 / d$. Indeed: (i) By Theorem 1.2 in [20] (see also Theorem 1.1 in [21]) we have $n^{1-1 / d}=O\left(\operatorname{Var}\left(H_{n}\right)\right)$. (ii) One can show that the functionals $H_{n}, n \geq 1$, are stabilizing following the proof of Theorem 3.4 in [8] (and the references cited therein). For the sake of completeness, we briefly sketch the line of the proof. For $n \geq 1$ and $x \in \eta_{n} \cap \mathbb{Y}$, we set

$$
\xi\left(x, \eta_{n} \cap \mathbb{Y}\right):=\mathbf{1}_{A}(x) \ell_{d}\left(\mathrm{C}\left(x, \eta_{n} \cap \mathbb{Y}\right) \cap A^{c}\right)-\mathbf{1}_{A^{c}}(x) \ell_{d}\left(\mathrm{C}\left(x, \eta_{n} \cap \mathbb{Y}\right) \cap A\right)
$$


and $\xi_{n}\left(x, \eta_{n} \cap \mathbb{Y}\right):=n \xi\left(x, \eta_{n} \cap \mathbb{Y}\right)$. It turns out that $H_{n}=\sum_{x \in \eta_{n} \cap \mathbb{Y}} \xi_{n}\left(x, \eta_{n} \cap \mathbb{Y}\right)$ and that the score functions $\xi_{n}, n \geq 1$, satisfy (2.3), (2.4) and (2.5) with $K:=\partial A$. (iii) To prove $I_{K, n}=I_{\partial A, n}=$ $O\left(n^{1-1 / d}\right)$, for the sake of clarity, we reproduce the argument in the proof of Theorem 2.3 in 8$]$. For all the notions and results of geometric measure theory considered hereon we refer the reader to [1]. We preliminary note that since $A$ is a convex body, it has a $(d-1)$-rectifiable boundary (i.e., its boundary is the Lipschitz image of a bounded set in $\left.\mathbb{R}^{d-1}\right)$. Then the $(d-1)$-dimensional upper Minkowski content of $\partial A$, denoted by $\overline{\mathcal{M}}^{d-1}(\partial A)$, is a scalar multiple of the $(d-1)$-dimensional Hausdorff measure of $\partial A$, denoted by $\mathcal{H}^{d-1}(\partial A)$. Therefore $\overline{\mathcal{M}}^{d-1}(\partial A)<\infty$, and by Lemma 5.12 in [8], there exists a positive constant $C>0$ such that

$$
\mathcal{H}^{d-1}\left(\partial A_{r}\right) \leq C\left(1+r^{d-1}\right), \quad r>0
$$

where $\partial A_{r}:=\left\{x \in \mathbb{R}^{d}: \mathrm{d}(x, \partial A) \leq r\right\}$. Setting $c:=p^{\prime \prime} c_{p^{\prime}} /\left[2^{2 \alpha+3}\left(4+p^{\prime \prime}\right)\right]$, we finally have

$$
\begin{aligned}
I_{K, n} & =n \int_{\mathbb{Y}} \exp \left(-c n^{\alpha / d} \mathrm{~d}(x, \partial A)^{\alpha}\right) \mathrm{d} x \\
& =n \int_{\mathbb{Y} \backslash \partial A} \exp \left(-c n^{\alpha / d} \mathrm{~d}(x, \partial A)^{\alpha}\right) \mathrm{d} x \\
& =n \int_{0}^{\infty} \exp \left(-c n^{\alpha / d} r^{\alpha}\right) \mathcal{H}^{d-1}\left(\partial A_{r}\right) \mathrm{d} r \\
& \leq C n \int_{0}^{\infty} \exp \left(-c n^{\alpha / d} r^{\alpha}\right)\left(1+r^{d-1}\right) \mathrm{d} r \\
& \leq C n^{1-1 / d} \int_{0}^{\infty} \mathrm{e}^{-c u^{\alpha}}\left(1+u^{d-1}\right) \mathrm{d} u,
\end{aligned}
$$

where (4.2) follows by the coarea formula and (4.3) is a consequence of (4.1).

\section{Proof of Theorem 2.6}

The proof of Theorem 2.6 relies on some inequalities for Malliavin's operators on the Poisson space recently derived by Last, Peccati and Schulte [11. We also exploit a general characterization of the almost sure version of the classical weak convergence of random variables due to Ibragimov and Lifshits [7]. In Subsection 5.1 we provide some further preliminaries about the Malliavin calculus on the Poisson space, in Subsection 5.2 we give the preliminary lemmas which will be exploited to prove Theorem 2.6 and finally in Subsection 5.3 we prove the theorem.

\subsection{Elements of the Malliavin calculus on the Poisson space}

For $r \in \mathbb{R}_{+}$and $n \geq 1$ integer, we denote by $L^{r}\left(\mu^{\otimes n}\right)$ the set of all measurable functions $g$ : $\mathbb{X}^{n} \rightarrow \mathbb{R}$ such that $\int_{\mathbb{X}^{n}}|g(x)|^{r} \mu^{\otimes n}(\mathrm{~d} x)<\infty$. We call a function $g: \mathbb{X}^{n} \rightarrow \mathbb{R}$ symmetric if it is 
invariant under permutations of its arguments, and denote by $L_{s}^{2}\left(\mu^{\otimes n}\right)$ the set of all symmetric functions $g \in L^{2}\left(\mu^{\otimes n}\right)$. For $g_{1}, g_{2} \in L^{2}\left(\mu^{\otimes n}\right)$, we define $\left\langle g_{1}, g_{2}\right\rangle_{n}:=\int_{\mathbb{X}^{n}} g_{1}(x) g_{2}(x) \mu^{\otimes n}(\mathrm{~d} x)$ and $\left\|g_{1}\right\|_{n}:=\sqrt{\left\langle g_{1}, g_{1}\right\rangle_{n}}$.

For $F \in L_{\eta}^{2}, F=f(\eta)$, we extend the definition of the Malliavin operators $D$ and $D^{2}$ defining $D_{x_{1}, \ldots, x_{n}}^{n} F:=D_{x_{n}}\left(D_{x_{1}, \ldots, x_{n-1}}^{n-1} F\right), x_{1}, \ldots, x_{n} \in \mathbb{X}, n \geq 3$. It is well-known that every $F \in L_{\eta}^{2}$ admits the representation

$$
F=\mathbb{E}[F]+\sum_{n \geq 1} I_{n}\left(g_{n}\right)
$$

where $g_{n}\left(x_{1}, \ldots, x_{n}\right)=\frac{1}{n !} \mathbb{E}\left[D_{x_{1}, \ldots, x_{n}}^{n} F\right]$ and, for $g_{n} \in L_{s}^{2}\left(\mu^{\otimes n}\right)$, we denote by $I_{n}\left(g_{n}\right)$ the $n$th order Wiener-Itô integral with respect to the centered Poisson measure $\eta(\mathrm{d} x)-\mu(\mathrm{d} x)$, see e.g. [9]. Another operator that we shall consider is the so-called Ornstein-Uhlenbeck generator $L$. Given $F \in L_{\eta}^{2}$ of the form (5.1), we write $F \in \operatorname{Dom}(L)$ if $\sum_{n \geq 1} n^{2} n !\left\|g_{n}\right\|_{n}^{2}<\infty$. In this case we define

$$
L F:=-\sum_{n \geq 1} n I_{n}\left(g_{n}\right)
$$

The (pseudo) inverse of $L$ is given by

$$
L^{-1} F:=-\sum_{n \geq 1} n^{-1} I_{n}\left(g_{n}\right)
$$

It can be easily checked that the random variable $L^{-1} F$ is a well-defined element of $L_{\eta}^{2}$ for every $F \in L_{\eta}^{2}$. In the following, we write $F \in \operatorname{Dom}(D)$ if $F \in L_{\eta}^{2}$ and $\int_{\mathbb{X}} \mathbb{E}\left[\left|D_{x} F\right|^{2}\right] \mu(\mathrm{d} x)<\infty$.

\subsection{Preliminary lemmas}

In this subsection we provide some relations among Malliavin's operators on the Poisson space, which will be crucial to prove Theorem 2.6. The following lemmas hold.

Lemma 5.1 For $F \in L_{\eta}^{2}$ and $r \geq 1$, we have

$$
\mathbb{E}\left[\left|D_{x} L^{-1} F\right|^{r}\right] \leq \mathbb{E}\left[\left|D_{x} F\right|^{r}\right], \quad \text { н-a.e. } x \in \mathbb{X}
$$

Lemma 5.2 For any $F, G \in \operatorname{Dom}(D)$ such that $\mathbb{E}[F]=0$, we have

$$
\mathbb{E}[F G]=\mathbb{E}\left[\left\langle D G,-D L^{-1} F\right\rangle_{1}\right]
$$

Lemma 5.3 For $F \in \operatorname{Dom}(D)$ such that $\mathbb{E}[F]=0$, we have

$$
\operatorname{Var}\left(\left\langle D F,-D L^{-1} F\right\rangle_{1}\right) \leq \gamma_{1}(F)^{2}
$$


where

$$
\begin{gathered}
\gamma_{1}(F)^{2}:=4 \int_{\mathbb{X}^{3}}\left(\mathbb{E}\left[\left(D_{x_{1}, x_{3}}^{2} F\right)^{2}\left(D_{x_{2}, x_{3}}^{2} F\right)^{2}\right]\right)^{1 / 2}\left(\mathbb{E}\left[\left(D_{x_{1}} F\right)^{2}\left(D_{x_{2}} F\right)^{2}\right]\right)^{1 / 2} \mu^{\otimes 3}\left(\mathrm{~d} x_{1}, \mathrm{~d} x_{2}, \mathrm{~d} x_{3}\right) \\
+\int_{\mathbb{X}^{3}} \mathbb{E}\left[\left(D_{x_{1}, x_{3}}^{2} F\right)^{2}\left(D_{x_{2}, x_{3}}^{2} F\right)^{2}\right] \mu^{\otimes 3}\left(\mathrm{~d} x_{1}, \mathrm{~d} x_{2}, \mathrm{~d} x_{3}\right) .
\end{gathered}
$$

We refer the reader to [11] for the proof of these lemmas (see, respectively, Lemma 3.4, the first displayed formula in the proof of Proposition 4.1 and the statement of Proposition 4.1 itself, therein).

The next lemma gives a Gaussian bound for functionals of the Poisson measure. We refer the reader to Lemma 2.2 in [3] and Lemma 3.1 in [24] for analogous inequalities on the Wiener and Rademacher spaces, respectively.

Lemma 5.4 For $F \in \operatorname{Dom}(D)$ such that $\mathbb{E}[F]=0$, we have

$$
\left|\mathbb{E}\left[\mathrm{e}^{\mathrm{i} t F}\right]-\mathrm{e}^{-t^{2} / 2}\right| \leq|t|^{2}\left(\left|1-\mathbb{E}\left[F^{2}\right]\right|+\gamma_{1}(F)\right)+\frac{|t|^{3}}{\sqrt{2}} \gamma_{2}(F), \quad \forall t \in \mathbb{R}
$$

where $\mathbf{i}:=\sqrt{-1}$ and

$$
\gamma_{2}(F):=\int_{\mathbb{X}} \mathbb{E}\left[\left|D_{x} F\right|^{3}\right] \mu(\mathrm{d} x)
$$

Proof. Let $\mathcal{C}_{b}^{1}(\mathbb{R})$ be the family of bounded and differentiable functions from $\mathbb{R}$ to $\mathbb{R}$ with a bounded first derivative. For any $g \in \mathcal{C}_{b}^{1}(\mathbb{R})$ it holds $g(F) \in \operatorname{Dom}(D)$ (indeed, we clearly have $g(F) \in L_{\eta}^{2}$ and, by the mean value theorem, $\left|D_{x} g(F)\right| \leq\left\|g^{\prime}\right\|_{\infty}\left|D_{x} F\right|$, for any $\left.x \in \mathbb{X}\right)$. Therefore, by Lemma 5.2

$$
\mathbb{E}[F g(F)]=\mathbb{E}\left[\left\langle D g(F),-D L^{-1} F\right\rangle_{1}\right], \quad \forall g \in \mathcal{C}_{b}^{1}(\mathbb{R})
$$

So, for any $t \in \mathbb{R}$,

$$
\begin{aligned}
\mathbb{E}\left[F \mathrm{e}^{\mathrm{i} t F}\right] & =\mathbb{E}[F \cos (t F)]+\mathbf{i} \mathbb{E}[F \sin (t F)] \\
& =\mathbb{E}\left[\left\langle D \cos (t F),-D L^{-1} F\right\rangle_{1}\right]+\mathbf{i} \mathbb{E}\left[\left\langle D \sin (t F),-D L^{-1} F\right\rangle_{1}\right]
\end{aligned}
$$

For $F=f(\eta)$, by Taylor's formula with integral remainder, for $(x, t) \in \mathbb{X} \times \mathbb{R}$,

$$
D_{x} \cos (t F)=\cos \left(t f\left(\eta+\varepsilon_{x}\right)\right)-\cos (t f(\eta))=-t \sin (t F) D_{x} F+t^{2} R_{1, t}(x)
$$

and similarly

$$
D_{x} \sin (t F)=\sin \left(t f\left(\eta+\varepsilon_{x}\right)\right)-\sin (t f(\eta))=t \cos (t F) D_{x} F+t^{2} R_{2, t}(x),
$$

where

$$
R_{1, t}(x):=\int_{f(\eta)}^{f\left(\eta+\varepsilon_{x}\right)}\left(u-f\left(\eta+\varepsilon_{x}\right)\right) \cos t u \mathrm{~d} u
$$


and

$$
R_{2, t}(x):=\int_{f(\eta)}^{f\left(\eta+\varepsilon_{x}\right)}\left(u-f\left(\eta+\varepsilon_{x}\right)\right) \sin t u \mathrm{~d} u .
$$

Note that, for $i=1,2,\left|R_{i, t}(x)\right| \leq \frac{\left|D_{x} F\right|^{2}}{2}$ and so

$$
\left|R_{1, t}(x)+\mathbf{i} R_{2, t}(x)\right| \leq \frac{\left|D_{x} F\right|^{2}}{\sqrt{2}}
$$

By (5.4), (5.5) and (5.6), we have

$$
\begin{aligned}
\mathbb{E}\left[F \mathrm{e}^{\mathrm{i} t F}\right] & =t \mathbb{E}\left[(-\sin (t F)+\mathbf{i} \cos (t F))\left\langle D F,-D L^{-1} F\right\rangle_{1}\right]+t^{2} \mathbb{E}\left[\left\langle R_{1, t}+\mathbf{i} R_{2, t},-D L^{-1} F\right\rangle_{1}\right] \\
& =\mathbf{i} t \mathbb{E}\left[\mathrm{e}^{\mathbf{i} t F}\left\langle D F,-D L^{-1} F\right\rangle_{1}\right]+t^{2} \mathbb{E}\left[\left\langle R_{1, t}+\mathbf{i} R_{2, t},-D L^{-1} F\right\rangle_{1}\right] .
\end{aligned}
$$

We put $\varphi(t):=\mathrm{e}^{t^{2} / 2} \mathbb{E}\left[\mathrm{e}^{\mathrm{i} t F}\right], t \in \mathbb{R}$. By the mean value theorem, (5.8) and (5.7), we have

$$
\begin{aligned}
&|\varphi(t)-\varphi(0)| \leq|t| \sup _{u \in[0, t]}\left|\varphi^{\prime}(u)\right| \\
&=|t| \sup _{u \in[0, t]}\left|u \mathrm{e}^{u^{2} / 2} \mathbb{E}\left[\mathrm{e}^{\mathbf{i} u F}\right]+\mathbf{i} \mathrm{e}^{u^{2} / 2} \mathbb{E}\left[F \mathrm{e}^{\mathbf{i} u F}\right]\right| \\
&=|t| \sup _{u \in[0, t]} \mid u \mathrm{e}^{u^{2} / 2} \mathbb{E}\left[\mathrm{e}^{\mathbf{i} u F}\right]-u \mathrm{e}^{u^{2} / 2} \mathbb{E}\left[\mathrm{e}^{\mathbf{i} u F}\left\langle D F,-D L^{-1} F\right\rangle_{1}\right] \\
& \quad+\mathbf{i} u^{2} \mathrm{e}^{u^{2} / 2} \mathbb{E}\left[\left\langle R_{1, u}+\mathbf{i} R_{2, u},-D L^{-1} F\right\rangle_{1}\right] \mid \\
& \leq|t|^{2} \mathrm{e}^{t^{2} / 2} \sup _{u \in[0, t]}\left|\mathbb{E}\left[\mathrm{e}^{\mathbf{i} u F}\left(1-\left\langle D F,-D L^{-1} F\right\rangle_{1}\right)\right]\right| \\
& \quad+|t|^{3} \mathrm{e}^{t^{2} / 2} \sup _{u \in[0, t]} \mathbb{E}\left[\left|\left\langle R_{1, u}+\mathbf{i} R_{2, u},-D L^{-1} F\right\rangle_{1}\right|\right] \\
& \leq|t|^{2} \mathrm{e}^{t^{2} / 2} \mathbb{E}\left[\left|1-\left\langle D F,-D L^{-1} F\right\rangle_{1}\right|\right] \\
& \quad+\frac{|t|^{3} \mathrm{e}^{t^{2} / 2}}{\sqrt{2}} \int_{\mathbb{X}} \mathbb{E}\left[\left|D_{x} F\right|^{2}\left|D_{x} L^{-1} F\right|\right] \mu(\mathrm{d} x) .
\end{aligned}
$$

So by Lemma 5.2, the Cauchy-Schwarz inequality and Lemma 5.3, it follows

$$
\begin{gathered}
\left|\mathbb{E}\left[\mathrm{e}^{\mathrm{i} t F}\right]-\mathrm{e}^{-t^{2} / 2}\right| \leq|t|^{2}\left|1-\mathbb{E}\left[F^{2}\right]\right|+|t|^{2} \mathbb{E}\left[\left|\mathbb{E}\left[F^{2}\right]-\left\langle D F,-D L^{-1} F\right\rangle_{1}\right|\right] \\
+\frac{|t|^{3}}{\sqrt{2}} \int_{\mathbb{X}} \mathbb{E}\left[\left|D_{x} F\right|^{2}\left|D L^{-1} F\right|\right] \mu(\mathrm{d} x) \\
\leq|t|^{2}\left|1-\mathbb{E}\left[F^{2}\right]\right|+|t|^{2} \sqrt{\mathbb{V a r}\left(\left\langle D F,-D L^{-1} F\right\rangle_{1}\right)} \\
+\frac{|t|^{3}}{\sqrt{2}} \int_{\mathbb{X}} \mathbb{E}\left[\left|D_{x} F\right|^{2}\left|D_{x} L^{-1} F\right|\right] \mu(\mathrm{d} x) \\
\leq|t|^{2}\left(\left|1-\mathbb{E}\left[F^{2}\right]\right|+\gamma_{1}(F)\right)+\frac{|t|^{3}}{\sqrt{2}} \int_{\mathbb{X}} \mathbb{E}\left[\left|D_{x} F\right|^{2}\left|D_{x} L^{-1} F\right|\right] \mu(\mathrm{d} x) .
\end{gathered}
$$

By Hölder's inequality and Lemma 5.1, we have

$$
\int_{X} \mathbb{E}\left[\left|D_{x} F\right|^{2}\left|D_{x} L^{-1} F\right|\right] \mu(\mathrm{d} x) \leq \int_{\mathbb{X}} \mathbb{E}\left[\left|D_{x} F\right|^{3}\right]^{2 / 3} \mathbb{E}\left[\left|D_{x} L^{-1} F\right|^{3}\right]^{1 / 3} \mu(\mathrm{d} x)
$$




$$
\begin{aligned}
& \leq \int_{\mathbb{X}} \mathbb{E}\left[\left|D_{x} F\right|^{3}\right]^{2 / 3} \mathbb{E}\left[\left|D_{x} F\right|^{3}\right]^{1 / 3} \mu(\mathrm{d} x) \\
& =\int_{\mathbb{X}} \mathbb{E}\left[\left|D_{x} F\right|^{3}\right] \mu(\mathrm{d} x) .
\end{aligned}
$$

The claim follows combining this latter inequality with (5.9).

We conclude this subsection recalling a result, due to Ibragimov and Lifshits [7], which provides general sufficient conditions for the ASCLT to hold.

Lemma 5.5 Let $\left\{X_{n}\right\}_{n \geq 1}$ be a sequence of real-valued random variables converging in distribution to a random variable $X$, and put

$$
\Delta_{n}(t):=\frac{1}{\log n} \sum_{k=1}^{n} \frac{1}{k}\left(\mathrm{e}^{\mathrm{i} t X_{k}}-\mathbb{E}\left[\mathrm{e}^{\mathrm{i} t X}\right]\right), \quad t \in \mathbb{R} .
$$

If, for all $r \in \mathbb{R}_{+}$,

$$
\sup _{|t| \leq r} \sum_{n \geq 2} \frac{\mathbb{E}\left[\left|\Delta_{n}(t)\right|^{2}\right]}{n \log n}<\infty
$$

then (1.1) holds with $X$ in place of $Z$. If $X$ is distributed according to the standard normal law, then $\left\{X_{n}\right\}_{n \geq 1}$ satisfies the ASCLT.

\subsection{Ancillary ASCLTs on the Poisson space and proof of Theorem 2.6}

Let $F_{1}, F_{2}, F_{3}, F_{4} \in L_{\eta}^{2}$ be such that $\mathbb{E}\left[F_{i}\right]=0, i=1,2,3,4$. We generalize the definition of the quantity $\gamma_{1}(F)^{2}$ setting

$$
\gamma\left(F_{1}, F_{2}, F_{3}, F_{4}\right)^{2}:=4 \lambda\left(F_{1}, F_{2}, F_{3}, F_{4}\right)^{2}+\int_{\mathbb{X}^{3}} \mathbb{E}\left[\left(D_{x_{1}, x_{3}}^{2} F_{1}\right)^{2}\left(D_{x_{2}, x_{3}}^{2} F_{2}\right)^{2}\right] \mu^{\otimes 3}\left(\mathrm{~d} x_{1}, \mathrm{~d} x_{2}, \mathrm{~d} x_{3}\right),
$$

where

$\lambda\left(F_{1}, F_{2}, F_{3}, F_{4}\right)^{2}:=\int_{\mathbb{X}^{3}}\left(\mathbb{E}\left[\left(D_{x_{1}, x_{3}}^{2} F_{1}\right)^{2}\left(D_{x_{2}, x_{3}}^{2} F_{2}\right)^{2}\right]\right)^{1 / 2}\left(\mathbb{E}\left[\left(D_{x_{1}} F_{3}\right)^{2}\left(D_{x_{2}} F_{4}\right)^{2}\right]\right)^{1 / 2} \mu^{\otimes 3}\left(\mathrm{~d} x_{1}, \mathrm{~d} x_{2}, \mathrm{~d} x_{3}\right)$.

Note that $\gamma_{1}\left(F_{1}\right)^{2}=\gamma\left(F_{1}, F_{1}, F_{1}, F_{1}\right)^{2}$,

$$
\lambda\left(F_{1}, F_{2}, F_{3}, F_{4}\right)^{2}=\lambda\left(F_{2}, F_{1}, F_{4}, F_{3}\right)^{2} \quad \text { and } \quad \gamma\left(F_{1}, F_{2}, F_{3}, F_{4}\right)^{2}=\gamma\left(F_{2}, F_{1}, F_{4}, F_{3}\right)^{2} .
$$

We also put

$$
\theta\left(F_{1}, F_{2}\right):=\int_{\mathbb{X}}\left(\mathbb{E}\left[\left|D_{x} F_{1}\right|^{2}\right]\right)^{1 / 2}\left(\mathbb{E}\left[\left|D_{x} F_{2}\right|^{2}\right]\right)^{1 / 2} \mu(\mathrm{d} x) .
$$

The following ASCLT will be proved at the end of this subsection. 
Proposition 5.6 Assume $\left\{F_{n}\right\}_{n \geq 1} \subset L_{\eta}^{2}$ and $\mathbb{E}\left[F_{n}\right]=0, \mathbb{E}\left[F_{n}^{2}\right]=1$, for any $n \geq 1$. Moreover, suppose

$$
\begin{gathered}
\lim _{n \rightarrow \infty} \gamma_{1}\left(F_{n}\right)=\lim _{n \rightarrow \infty} \gamma_{2}\left(F_{n}\right)=0, \\
\sum_{n \geq 2} \frac{1}{n(\log n)^{2}} \sum_{k=1}^{n} \frac{\gamma_{i}\left(F_{k}\right)}{k}<\infty, \quad i=1,2 \\
\sum_{n \geq 2} \frac{1}{n(\log n)^{3}} \sum_{l=1}^{n} \sum_{k=1}^{l} \frac{\theta\left(F_{k}, F_{l}\right)}{k l}<\infty \\
\sum_{n \geq 2} \frac{1}{n(\log n)^{3}} \sum_{l=1}^{n} \sum_{k=1}^{l} \frac{\gamma\left(F_{k}, F_{l}, F_{k}, F_{l}\right)}{k l}<\infty \\
\sum_{n \geq 2} \frac{1}{n(\log n)^{3}} \sum_{l=1}^{n} \sum_{k=1}^{l} \frac{\lambda_{\max }\left(F_{k}, F_{l}\right)}{k l}<\infty
\end{gathered}
$$

where

$$
\begin{aligned}
\lambda_{\max }\left(F_{k}, F_{l}\right):= & \lambda\left(F_{k}, F_{k}, F_{k}, F_{l}\right) \vee \lambda\left(F_{l}, F_{l}, F_{l}, F_{k}\right) \vee \lambda\left(F_{k}, F_{k}, F_{l}, F_{l}\right) \vee \lambda\left(F_{l}, F_{l}, F_{k}, F_{k}\right) \\
& \vee \lambda\left(F_{l}, F_{k}, F_{k}, F_{k}\right) \vee \lambda\left(F_{k}, F_{l}, F_{l}, F_{l}\right) \vee \lambda\left(F_{k}, F_{l}, F_{l}, F_{k}\right) .
\end{aligned}
$$

Then $\left\{F_{n}\right\}_{n \geq 1}$ satisfies the ASCLT.

Next, to state a corollary of this proposition, we introduce some more notation. For $F_{1}, F_{2}, F_{3}, F_{4} \in$ $L_{\eta}^{2}$ such that $\mathbb{E}\left[F_{i}\right]=0, i=1,2,3,4$, we set

$$
\begin{gathered}
\Gamma_{1}\left(F_{1}\right)^{2}:=4 \int_{\mathbb{X}^{3}} \mathbb{E}\left[\left(D_{x_{1}, x_{3}}^{2} F_{1}\right)^{4}\right]^{1 / 4} \mathbb{E}\left[\left(D_{x_{2}, x_{3}}^{2} F_{1}\right)^{4}\right]^{1 / 4} \mathbb{E}\left[\left(D_{x_{1}} F_{1}\right)^{4}\right]^{1 / 4} \mathbb{E}\left[\left(D_{x_{2}} F_{1}\right)^{4}\right]^{1 / 4} \mu^{\otimes 3}\left(\mathrm{~d} x_{1}, \mathrm{~d} x_{2}, \mathrm{~d} x_{3}\right) \\
+\int_{\mathbb{X}^{3}} \mathbb{E}\left[\left(D_{x_{1}, x_{3}}^{2} F_{1}\right)^{4}\right]^{1 / 2} \mathbb{E}\left[\left(D_{x_{2}, x_{3}}^{2} F_{1}\right)^{4}\right]^{1 / 2} \mu^{\otimes 3}\left(\mathrm{~d} x_{1}, \mathrm{~d} x_{2}, \mathrm{~d} x_{3}\right) \\
=4 \int_{\mathbb{X}}\left(\int_{\mathbb{X}} \mathbb{E}\left[\left(D_{x_{1}, x_{2}}^{2} F_{1}\right)^{4}\right]^{1 / 4} \mathbb{E}\left[\left(D_{x_{1}} F_{1}\right)^{4}\right]^{1 / 4} \mu\left(\mathrm{d} x_{1}\right)\right)^{2} \mu\left(\mathrm{d} x_{2}\right) \\
+\int_{\mathbb{X}}\left(\int_{\mathbb{X}} \mathbb{E}\left[\left(D_{x_{1}, x_{2}}^{2} F_{1}\right)^{4}\right]^{1 / 2} \mu\left(\mathrm{d} x_{1}\right)\right)^{2} \mu\left(\mathrm{d} x_{2}\right), \\
\Gamma\left(F_{1}, F_{2}, F_{3}, F_{4}\right)^{2}:=4 \Lambda\left(F_{1}, F_{2}, F_{3}, F_{4}\right)^{2}+\int_{\mathbb{X}^{3}} \mathbb{E}\left[\left(D_{x_{1}, x_{3}}^{2} F_{1}\right)^{4}\right]^{1 / 2} \mathbb{E}\left[\left(D_{x_{2}, x_{3}}^{2} F_{2}\right)^{4}\right]^{1 / 2} \mu^{\otimes 3}\left(\mathrm{~d} x_{1}, \mathrm{~d} x_{2}, \mathrm{~d} x_{3}\right) \\
=4 \Lambda\left(F_{1}, F_{2}, F_{3}, F_{4}\right)^{2} \\
+\int_{\mathbb{X}}\left(\int_{\mathbb{X}} \mathbb{E}\left[\left(D_{x_{1}, x_{2}}^{2} F_{1}\right)^{4}\right]^{1 / 2} \mu\left(\mathrm{d} x_{1}\right)\right)\left(\int_{\mathbb{X}} \mathbb{E}\left[\left(D_{x_{1}, x_{2}}^{2} F_{2}\right)^{4}\right]^{1 / 2} \mu\left(\mathrm{d} x_{1}\right)\right) \mu\left(\mathrm{d} x_{2}\right),
\end{gathered}
$$

$\Lambda\left(F_{1}, F_{2}, F_{3}, F_{4}\right)^{2}$ 


$$
\begin{aligned}
: & =\int_{\mathbb{X}^{3}} \mathbb{E}\left[\left(D_{x_{1}, x_{3}}^{2} F_{1}\right)^{4}\right]^{1 / 4} \mathbb{E}\left[\left(D_{x_{2}, x_{3}}^{2} F_{2}\right)^{4}\right]^{1 / 4} \mathbb{E}\left[\left(D_{x_{1}} F_{3}\right)^{4}\right]^{1 / 4} \mathbb{E}\left[\left(D_{x_{2}} F_{4}\right)^{4}\right]^{1 / 4} \mu^{\otimes 3}\left(\mathrm{~d} x_{1}, \mathrm{~d} x_{2}, \mathrm{~d} x_{3}\right) \\
& =\int_{\mathbb{X}}\left(\int_{\mathbb{X}} \mathbb{E}\left[\left(D_{x_{1}, x_{2}}^{2} F_{1}\right)^{4}\right]^{1 / 4} \mathbb{E}\left[\left(D_{x_{1}} F_{3}\right)^{4}\right]^{1 / 4} \mu\left(\mathrm{d} x_{1}\right)\right)\left(\int_{\mathbb{X}} \mathbb{E}\left[\left(D_{x_{1}, x_{2}}^{2} F_{2}\right)^{4}\right]^{1 / 4} \mathbb{E}\left[\left(D_{x_{1}} F_{4}\right)^{4}\right]^{1 / 4} \mu\left(\mathrm{d} x_{1}\right)\right) \mu\left(\mathrm{d} x_{2}\right)
\end{aligned}
$$

and

$$
\Theta\left(F_{1}, F_{2}\right):=\int_{\mathbb{X}} \mathbb{E}\left[\left(D_{x} F_{1}\right)^{4}\right]^{1 / 4} \mathbb{E}\left[\left(D_{x} F_{2}\right)^{4}\right]^{1 / 4} \mu(\mathrm{d} x) .
$$

Note that $\Gamma_{1}\left(F_{1}\right)^{2}=\Gamma\left(F_{1}, F_{1}, F_{1}, F_{1}\right)^{2}$,

$$
\Lambda\left(F_{1}, F_{2}, F_{3}, F_{4}\right)^{2}=\Lambda\left(F_{2}, F_{1}, F_{4}, F_{3}\right)^{2} \quad \text { and } \quad \Gamma\left(F_{1}, F_{2}, F_{3}, F_{4}\right)^{2}=\Gamma\left(F_{2}, F_{1}, F_{4}, F_{3}\right)^{2} .
$$

Note also that by the Cauchy-Schwarz inequality

$\gamma\left(F_{1}, F_{2}, F_{3}, F_{4}\right)^{2} \leq \Gamma\left(F_{1}, F_{2}, F_{3}, F_{4}\right)^{2}, \quad \lambda\left(F_{1}, F_{2}, F_{3}, F_{4}\right)^{2} \leq \Lambda\left(F_{1}, F_{2}, F_{3}, F_{4}\right)^{2}, \quad \theta\left(F_{1}, F_{2}\right) \leq \Theta\left(F_{1}, F_{2}\right)$

(and, so, in particular, $\gamma_{1}\left(F_{1}\right) \leq \Gamma_{1}\left(F_{1}\right)$ ).

The following corollary is an immediate consequence of the inequalities (5.18) and Proposition 5.6 .

Corollary 5.7 Assume $\left\{F_{n}\right\}_{n \geq 1} \subset L_{\eta}^{2}$ and $\mathbb{E}\left[F_{n}\right]=0, \mathbb{E}\left[F_{n}^{2}\right]=1$, for any $n \geq 1$. Moreover, suppose (5.12) and (5.13) with $\Gamma_{1}$ in place of $\gamma_{1}$, (5.14) with $\Theta$ in place of $\theta$, (5.15) with $\Gamma$ in place of $\gamma$, and (5.16) with $\Lambda$ in place of $\lambda$. Then $\left\{F_{n}\right\}_{n \geq 1}$ satisfies the ASCLT.

Proof of Theorem [2.6. For $n \geq 1, x_{1}, x_{2} \in \mathbb{X}$, we have

$$
D_{x_{1}} F_{n}=\frac{D_{x_{1}} H_{n}}{\sqrt{\operatorname{Var}\left(H_{n}\right)}} \quad \text { and } \quad D_{x_{1}, x_{2}}^{2} F_{n}=\frac{D_{x_{1}, x_{2}}^{2} H_{n}}{\sqrt{\operatorname{Var}\left(H_{n}\right)}} .
$$

So, letting $C \in \mathbb{R}_{+}$denote a positive constant which may vary from line to line, by Hölder's inequality and assumptions (2.11) and (2.12), for any $n \geq 1$, we have

$$
\begin{aligned}
& \mathbb{E}\left[\left|D_{x_{1}} F_{n}\right|^{4}\right] \leq \mathbb{P}\left(D_{x_{1}} H_{n} \neq 0\right)^{p /(4+p)} \frac{\mathbb{E}\left[\left|D_{x_{1}} H_{n}\right|^{4+p}\right]^{4 /(4+p)}}{\operatorname{Var}\left(H_{n}\right)^{2}} \\
& \leq C \frac{\mathbb{P}\left(D_{x_{1}} H_{n} \neq 0\right)^{p /(4+p)}}{\operatorname{Var}\left(H_{n}\right)^{2}}, \quad \mu \text {-a.e. } x_{1} \in \mathbb{X} \\
& \mathbb{E}\left[\left|D_{x_{1}, x_{2}}^{2} F_{n}\right|^{4}\right] \leq C \frac{\mathbb{P}\left(D_{x_{1}, x_{2}}^{2} H_{n} \neq 0\right)^{q /(4+q)}}{\mathbb{V a r}\left(H_{n}\right)^{2}}, \quad \mu^{\otimes 2} \text {-a.e. }\left(x_{1}, x_{2}\right) \in \mathbb{X}^{2}
\end{aligned}
$$

and

$$
\mathbb{E}\left[\left|D_{x_{1}} F_{n}\right|^{3}\right] \leq \mathbb{P}\left(D_{x_{1}} H_{n} \neq 0\right)^{(1+p) /(4+p)} \frac{\mathbb{E}\left[\left|D_{x_{1}} H_{n}\right|^{4+p}\right]^{3 /(4+p)}}{\operatorname{Var}\left(H_{n}\right)^{3 / 2}}
$$




$$
\leq C \frac{\mathbb{P}\left(D_{x_{1}} H_{n} \neq 0\right)^{(1+p) /(4+p)}}{\operatorname{Var}\left(H_{n}\right)^{3 / 2}}, \quad \mu \text {-a.e. } x_{1} \in \mathbb{X} .
$$

For $k, l, m, n \geq 1$, the inequalities (5.19), (5.20) and (5.21) imply

$$
\begin{gathered}
\Gamma_{1}\left(F_{k}\right)^{2} \leq C \boldsymbol{\Gamma}_{1}\left(H_{k}\right)^{2}, \quad \Gamma\left(F_{k}, F_{l}, F_{m}, F_{n}\right)^{2} \leq C \boldsymbol{\Gamma}\left(H_{k}, H_{l}, H_{m}, H_{n}\right)^{2}, \\
\Lambda\left(F_{k}, F_{l}, F_{m}, F_{n}\right)^{2} \leq C \boldsymbol{\Lambda}\left(H_{k}, H_{l}, H_{m}, H_{n}\right)^{2}, \quad \Theta\left(F_{k}, F_{l}\right)^{2} \leq C \boldsymbol{\Theta}\left(H_{k}, H_{l}\right)^{2}, \quad \gamma_{2}\left(F_{k}\right) \leq C \boldsymbol{\Gamma}_{2}\left(H_{k}\right) .
\end{gathered}
$$

The claim follows by these relations and Corollary [5.7.

Proof of Proposition 5.6. We start noticing that in fact $F_{n} \in \operatorname{Dom}(D), n \geq 1$. Indeed, by (5.14) it follows

$$
\int_{\mathbb{X}} \mathbb{E}\left[\left|D_{x} F\right|^{2}\right] \mu(\mathrm{d} x)=\theta\left(F_{n}, F_{n}\right)<\infty, \quad n \geq 1
$$

To prove the ASCLT we are going to apply the Ibragimov and Lifshits criterion stated as Lemma 5.5. So, let $\Delta_{n}(t)$ be given by (5.10) with $F_{k}$ in place of $X_{k}$ and $Z$ in place of $X$. We have

$$
\begin{aligned}
& \mathbb{E}\left[\left|\Delta_{n}(t)\right|^{2}\right]= \frac{1}{(\log n)^{2}} \sum_{k, l}^{1, n} \frac{1}{k l} \mathbb{E}\left[\left(\mathrm{e}^{\mathrm{i} t F_{k}}-\mathrm{e}^{-t^{2} / 2}\right)\left(\mathrm{e}^{-\mathrm{i} t F_{l}}-\mathrm{e}^{-t^{2} / 2}\right)\right] \\
&=\frac{1}{(\log n)^{2}} \sum_{k, l}^{1, n} \frac{1}{k l}\left[\left(\mathbb{E}\left[\mathrm{e}^{\mathrm{i} t\left(F_{k}-F_{l}\right)}\right]-\mathrm{e}^{-t^{2}}\right)-\mathrm{e}^{-t^{2} / 2}\left(\mathbb{E}\left[\mathrm{e}^{\mathrm{i} t F_{k}}\right]-\mathrm{e}^{-t^{2} / 2}\right)\right. \\
&\left.-\mathrm{e}^{-t^{2} / 2}\left(\mathbb{E}\left[\mathrm{e}^{-\mathrm{i} t F_{l}}\right]-\mathrm{e}^{-t^{2} / 2}\right)\right] .
\end{aligned}
$$

Therefore, for $r \in \mathbb{R}_{+}$,

$$
\begin{aligned}
\sup _{|t| \leq r} \sum_{n \geq 2} \frac{\mathbb{E}\left[\left|\Delta_{n}(t)\right|^{2}\right]}{n \log n} \leq & \sup _{|t| \leq r} \sum_{n \geq 2} \frac{1}{n(\log n)^{3}} \sum_{k, l}^{1, n} \frac{\left|\mathbb{E}\left[\mathrm{e}^{\mathrm{i} t\left(F_{k}-F_{l}\right)}\right]-\mathrm{e}^{-t^{2}}\right|}{k l} \\
& +\sup _{|t| \leq r} \sum_{n \geq 2} \frac{1}{n(\log n)^{3}} \sum_{l=1}^{n} \frac{1}{l} \sum_{k=1}^{n} \frac{\left|\mathbb{E}\left[\mathrm{e}^{\mathrm{i} t F_{k}}\right]-\mathrm{e}^{-t^{2} / 2}\right|}{k} \\
& +\sup _{|t| \leq r} \sum_{n \geq 2} \frac{1}{n(\log n)^{3}} \sum_{k=1}^{n} \frac{1}{k} \sum_{l=1}^{n} \frac{\left|\mathbb{E}\left[\mathrm{e}^{-\mathrm{i} t F_{l}}\right]-\mathrm{e}^{-t^{2} / 2}\right|}{l} .
\end{aligned}
$$

Let $t \in \mathbb{R}$ and $r \in \mathbb{R}_{+}$be such that $|t| \leq r$. Since $\mathbb{E}\left[F_{n}^{2}\right]=1$ for each $n$, by Lemma 5.4 we have

$$
\left|\mathbb{E}\left[\mathrm{e}^{\mathbf{i} F_{k} t}\right]-\mathrm{e}^{-t^{2} / 2}\right| \leq r^{2} \gamma_{1}\left(F_{k}\right)+\frac{r^{3}}{\sqrt{2}} \gamma_{2}\left(F_{k}\right) .
$$

Therefore,

$$
\sup _{|t| \leq r} \sum_{n \geq 2} \frac{1}{n(\log n)^{3}} \sum_{l=1}^{n} \frac{1}{l} \sum_{k=1}^{n} \frac{\left|\mathbb{E}\left[\mathrm{e}^{\mathrm{i} t F_{k}}\right]-\mathrm{e}^{-t^{2} / 2}\right|}{k} \leq \sum_{n \geq 2} \frac{1}{n(\log n)^{3}} \sum_{l=1}^{n} \frac{1}{l} \sum_{k=1}^{n} \frac{r^{2} \gamma_{1}\left(F_{k}\right)+2^{-1 / 2} r^{3} \gamma_{2}\left(F_{k}\right)}{k} .
$$


Since $\lim _{n \rightarrow \infty} \sum_{l=1}^{n} l^{-1} / \log n=1$, the finiteness of the term in (5.23) is guaranteed by the assumption (5.13). Since by Lemma 5.4 we also have

$$
\left|\mathbb{E}\left[\mathrm{e}^{-\mathbf{i} t F_{l}}\right]-\mathrm{e}^{-t^{2} / 2}\right| \leq r^{2} \gamma_{1}\left(F_{l}\right)+\frac{r^{3}}{\sqrt{2}} \gamma_{2}\left(F_{l}\right),
$$

the same argument guarantees the finiteness of the term in (5.24). As far as the finiteness of the term in the right-hand side of (5.22), we start noticing that, again by Lemma 5.4 we get

$$
\begin{aligned}
\left|\mathbb{E}\left[\mathrm{e}^{\mathrm{i} t\left(F_{k}-F_{l}\right)}\right]-\mathrm{e}^{-t^{2}}\right| & =\left|\mathbb{E}\left[\mathrm{e}^{\mathrm{i}(\sqrt{2} t)\left(F_{k}-F_{l}\right) / \sqrt{2}}\right]-\mathrm{e}^{-(\sqrt{2} t)^{2} / 2}\right| \\
& \leq 2|t|^{2}\left|\mathbb{E}\left[F_{k} F_{l}\right]\right|+2|t|^{2} \gamma_{1}\left(\frac{F_{k}-F_{l}}{\sqrt{2}}\right)+2|t|^{3} \gamma_{2}\left(\frac{F_{k}-F_{l}}{\sqrt{2}}\right) \\
& \leq 2 r^{2} \mathbb{E}\left[F_{k} F_{l}\right] \mid+2 r^{2} \gamma_{1}\left(\frac{F_{k}-F_{l}}{\sqrt{2}}\right)+2 r^{3} \gamma_{2}\left(\frac{F_{k}-F_{l}}{\sqrt{2}}\right) .
\end{aligned}
$$

Therefore the term in the right-hand side of $(5.22)$ is finite if

$$
\begin{aligned}
& \sum_{n \geq 2} \frac{1}{n(\log n)^{3}} \sum_{k, l}^{1, n} \frac{\left|\mathbb{E}\left[F_{k} F_{l}\right]\right|}{k l}<\infty, \\
& \sum_{n \geq 2} \frac{1}{n(\log n)^{3}} \sum_{k, l}^{1, n} \frac{\gamma_{2}\left(\frac{F_{k}-F_{l}}{\sqrt{2}}\right)}{k l}<\infty, \\
& \sum_{n \geq 2} \frac{1}{n(\log n)^{3}} \sum_{k, l}^{1, n} \frac{\gamma_{1}\left(\frac{F_{k}-F_{l}}{\sqrt{2}}\right)}{k l}<\infty .
\end{aligned}
$$

Proof of (5.25). Due to the symmetry (with respect to $k$ and $l$ ) of the mapping $(k, l) \mapsto \frac{\left|\mathbb{E}\left[F_{k} F_{l}\right]\right|}{k l}$, it holds

$$
\sum_{n \geq 2} \frac{1}{n(\log n)^{3}} \sum_{k, l}^{1, n} \frac{\left|\mathbb{E}\left[F_{k} F_{l}\right]\right|}{k l}=\sum_{n \geq 2} \frac{1}{n(\log n)^{3}} \sum_{k=1}^{n} \frac{1}{k^{2}}+2 \sum_{n \geq 2} \frac{1}{n(\log n)^{3}} \sum_{l=2}^{n} \sum_{k=1}^{l-1} \frac{\left|\mathbb{E}\left[F_{k} F_{l}\right]\right|}{k l}
$$

Since $\sum_{n \geq 2} \frac{1}{n(\log n)^{\beta}}<\infty, \beta>1$, and $\sum_{n \geq 2} \frac{1}{n^{2}}<\infty$, the infinite sum (5.25) converges if and only if

$$
\sum_{n \geq 2} \frac{1}{n(\log n)^{3}} \sum_{l=1}^{n} \sum_{k=1}^{l} \frac{\left|\mathbb{E}\left[F_{k} F_{l}\right]\right|}{k l}<\infty .
$$

By Lemmas 5.2, 5.1 and the Cauchy-Schwarz inequality, we get

$$
\begin{aligned}
\left|\mathbb{E}\left[F_{k} F_{l}\right]\right| & \leq \mathbb{E}\left[\left|\left\langle D F_{k},-D L^{-1} F_{l}\right\rangle_{1}\right|\right] \\
& \leq \int_{\mathbb{X}} \mathbb{E}\left[\left|D_{x} F_{k}\right|\left|D_{x} L^{-1} F_{l}\right|\right] \mu(\mathrm{d} x) \leq \theta\left(F_{k}, F_{l}\right),
\end{aligned}
$$

and therefore (5.28) is a consequence of the assumption (5.14).

Proof of (5.26). By the inequality $(a+b)^{3} \leq 4\left(a^{3}+b^{3}\right), a, b \geq 0$, we have

$$
\gamma_{2}\left(\frac{F_{k}-F_{l}}{\sqrt{2}}\right)=2^{-3 / 2} \int_{\mathbb{X}} \mathbb{E}\left[\left|D_{x} F_{k}-D_{x} F_{l}\right|^{3}\right] \mu(\mathrm{d} x)
$$




$$
\leq \sqrt{2}\left(\gamma_{2}\left(F_{k}\right)+\gamma_{2}\left(F_{l}\right)\right)
$$

Therefore, (5.26) follows by the assumption (5.13) (with $i=2$ ).

Proof of (5.27). Due to the symmetry (with respect to $k$ and $l$ ) of the mapping $(k, l) \mapsto \frac{\gamma_{1}\left(\frac{F_{k}-F_{l}}{\sqrt{2}}\right)}{k l}$, the infinite sum (5.27) converges if and only if

$$
\sum_{n \geq 2} \frac{1}{n(\log n)^{3}} \sum_{l=1}^{n} \sum_{k=1}^{l} \frac{\gamma_{1}\left(\frac{F_{k}-F_{l}}{\sqrt{2}}\right)}{k l}<\infty .
$$

Using the inequalities $(a+b)^{2} \leq 2 a^{2}+2 b^{2}$ and $(a+b)^{1 / 2} \leq a^{1 / 2}+b^{1 / 2}, a, b \geq 0$, we now bound the quantity $\gamma_{1}\left(\left(F_{k}-F_{l}\right) / \sqrt{2}\right)$. Hereon, for simplicity of notation we put $\mu^{\otimes 3}(\mathrm{~d} \mathbf{x}):=\mu^{\otimes 3}\left(\mathrm{~d} x_{1}, \mathrm{~d} x_{2}, \mathrm{~d} x_{3}\right)$. We have:

$$
\begin{aligned}
& \gamma_{1}\left(\frac{F_{k}-F_{l}}{\sqrt{2}}\right) \leq\left(4 \int_{\mathbb{X}^{3}}\left(\mathbb{E}\left[\left(\left|D_{x_{1}, x_{3}}^{2} F_{k}\right|^{2}+\left|D_{x_{1}, x_{3}}^{2} F_{l}\right|^{2}\right)\left(\left|D_{x_{2}, x_{3}}^{2} F_{k}\right|^{2}+\left|D_{x_{2}, x_{3}}^{2} F_{l}\right|^{2}\right)\right]\right)^{1 / 2}\right. \\
& \times\left(\mathbb{E}\left[\left(\left|D_{x_{1}} F_{k}\right|^{2}+\left|D_{x_{1}} F_{l}\right|^{2}\right)\left(\left|D_{x_{2}} F_{k}\right|^{2}+\left|D_{x_{2}} F_{l}\right|^{2}\right)\right]\right)^{1 / 2} \mu^{\otimes 3}(\mathrm{~d} \mathbf{x}) \\
& \left.+\int_{\mathbb{X}^{3}} \mathbb{E}\left[\left(\left|D_{x_{1}, x_{3}}^{2} F_{k}\right|^{2}+\left|D_{x_{1}, x_{3}}^{2} F_{l}\right|^{2}\right)\left(\left|D_{x_{2}, x_{3}}^{2} F_{k}\right|^{2}+\left|D_{x_{2}, x_{3}}^{2} F_{l}\right|^{2}\right)\right] \mu^{\otimes 3}(\mathrm{~d} \mathbf{x})\right)^{1 / 2} \\
& =\left(4 \int _ { \mathbb { X } ^ { 3 } } \left(\left(\mathbb{E}\left[\left|D_{x_{1}, x_{3}}^{2} F_{k}\right|^{2}\left|D_{x_{2}, x_{3}}^{2} F_{k}\right|^{2}\right]+\mathbb{E}\left[\left|D_{x_{1}, x_{3}}^{2} F_{k}\right|^{2}\left|D_{x_{2}, x_{3}}^{2} F_{l}\right|^{2}\right]\right.\right.\right. \\
& \left.+\mathbb{E}\left[\left|D_{x_{1}, x_{3}}^{2} F_{l}\right|^{2}\left|D_{x_{2}, x_{3}}^{2} F_{k}\right|^{2}\right]+\mathbb{E}\left[\left|D_{x_{1}, x_{3}}^{2} F_{l}\right|^{2}\left|D_{x_{2}, x_{3}}^{2} F_{l}\right|^{2}\right]\right) \\
& \times\left(\mathbb{E}\left[\left|D_{x_{1}} F_{k}\right|^{2}\left|D_{x_{2}} F_{k}\right|^{2}\right]+\mathbb{E}\left[\left|D_{x_{1}} F_{k}\right|^{2}\left|D_{x_{2}} F_{l}\right|^{2}\right]\right. \\
& \left.\left.+\mathbb{E}\left[\left|D_{x_{1}} F_{l}\right|^{2}\left|D_{x_{2}} F_{k}\right|^{2}\right]+\mathbb{E}\left[\left|D_{x_{1}} F_{l}\right|^{2}\left|D_{x_{2}} F_{l}\right|^{2}\right]\right)\right)^{1 / 2} \mu^{\otimes 3}(\mathrm{~d} \mathbf{x}) \\
& +\int_{\mathbb{X}^{3}}\left(\mathbb{E}\left[\left|D_{x_{1}, x_{3}}^{2} F_{k}\right|^{2}\left|D_{x_{2}, x_{3}}^{2} F_{k}\right|^{2}\right]+\mathbb{E}\left[\left|D_{x_{1}, x_{3}}^{2} F_{k}\right|^{2}\left|D_{x_{2}, x_{3}}^{2} F_{l}\right|^{2}\right]\right. \\
& \left.\left.+\mathbb{E}\left[\left|D_{x_{1}, x_{3}}^{2} F_{l}\right|^{2}\left|D_{x_{2}, x_{3}}^{2} F_{k}\right|^{2}\right]+\mathbb{E}\left[\left|D_{x_{1}, x_{3}}^{2} F_{l}\right|^{2}\left|D_{x_{2}, x_{3}}^{2} F_{l}\right|^{2}\right]\right) \mu^{\otimes 3}(\mathrm{~d} \mathbf{x})\right)^{1 / 2} \\
& =\left(4 \int_{\mathbb{X}^{3}} \mu^{\otimes 3}(\mathrm{~d} \mathbf{x})\right. \\
& \left(\mathbb{E}\left[\left|D_{x_{1}, x_{3}}^{2} F_{k}\right|^{2}\left|D_{x_{2}, x_{3}}^{2} F_{k}\right|^{2}\right] \mathbb{E}\left[\left|D_{x_{1}} F_{k}\right|^{2}\left|D_{x_{2}} F_{k}\right|^{2}\right]+\mathbb{E}\left[\left|D_{x_{1}, x_{3}}^{2} F_{k}\right|^{2}\left|D_{x_{2}, x_{3}}^{2} F_{k}\right|^{2}\right] \mathbb{E}\left[\left|D_{x_{1}} F_{k}\right|^{2}\left|D_{x_{2}} F_{l}\right|^{2}\right]\right. \\
& +\mathbb{E}\left[\left|D_{x_{1}, x_{3}}^{2} F_{k}\right|^{2}\left|D_{x_{2}, x_{3}}^{2} F_{k}\right|^{2}\right] \mathbb{E}\left[\left|D_{x_{1}} F_{l}\right|^{2}\left|D_{x_{2}} F_{k}\right|^{2}\right]+\mathbb{E}\left[\left|D_{x_{1}, x_{3}}^{2} F_{k}\right|^{2}\left|D_{x_{2}, x_{3}}^{2} F_{k}\right|^{2}\right] \mathbb{E}\left[\left|D_{x_{1}} F_{l}\right|^{2}\left|D_{x_{2}} F_{l}\right|^{2}\right] \\
& +\mathbb{E}\left[\left|D_{x_{1}, x_{3}}^{2} F_{k}\right|^{2}\left|D_{x_{2}, x_{3}}^{2} F_{l}\right|^{2}\right] \mathbb{E}\left[\left|D_{x_{1}} F_{k}\right|^{2}\left|D_{x_{2}} F_{k}\right|^{2}\right]+\mathbb{E}\left[\left|D_{x_{1}, x_{3}}^{2} F_{k}\right|^{2}\left|D_{x_{2}, x_{3}}^{2} F_{l}\right|^{2}\right] \mathbb{E}\left[\left|D_{x_{1}} F_{k}\right|^{2}\left|D_{x_{2}} F_{l}\right|^{2}\right] \\
& +\mathbb{E}\left[\left|D_{x_{1}, x_{3}}^{2} F_{k}\right|^{2}\left|D_{x_{2}, x_{3}}^{2} F_{l}\right|^{2}\right] \mathbb{E}\left[\left|D_{x_{1}} F_{l}\right|^{2}\left|D_{x_{2}} F_{k}\right|^{2}\right]+\mathbb{E}\left[\left|D_{x_{1}, x_{3}}^{2} F_{k}\right|^{2}\left|D_{x_{2}, x_{3}}^{2} F_{l}\right|^{2}\right] \mathbb{E}\left[\left|D_{x_{1}} F_{l}\right|^{2}\left|D_{x_{2}} F_{l}\right|^{2}\right] \\
& +\mathbb{E}\left[\left|D_{x_{1}, x_{3}}^{2} F_{l}\right|^{2}\left|D_{x_{2}, x_{3}}^{2} F_{k}\right|^{2}\right] \mathbb{E}\left[\left|D_{x_{1}} F_{k}\right|^{2}\left|D_{x_{2}} F_{k}\right|^{2}\right]+\mathbb{E}\left[\left|D_{x_{1}, x_{3}}^{2} F_{l}\right|^{2}\left|D_{x_{2}, x_{3}}^{2} F_{k}\right|^{2}\right] \mathbb{E}\left[\left|D_{x_{1}} F_{k}\right|^{2}\left|D_{x_{2}} F_{l}\right|^{2}\right] \\
& +\mathbb{E}\left[\left|D_{x_{1}, x_{3}}^{2} F_{l}\right|^{2}\left|D_{x_{2}, x_{3}}^{2} F_{k}\right|^{2}\right] \mathbb{E}\left[\left|D_{x_{1}} F_{l}\right|^{2}\left|D_{x_{2}} F_{k}\right|^{2}\right]+\mathbb{E}\left[\left|D_{x_{1}, x_{3}}^{2} F_{l}\right|^{2}\left|D_{x_{2}, x_{3}}^{2} F_{k}\right|^{2}\right] \mathbb{E}\left[\left|D_{x_{1}} F_{l}\right|^{2}\left|D_{x_{2}} F_{l}\right|^{2}\right]
\end{aligned}
$$




$$
\begin{aligned}
& +\mathbb{E}\left[\left|D_{x_{1}, x_{3}}^{2} F_{l}\right|^{2}\left|D_{x_{2}, x_{3}}^{2} F_{l}\right|^{2}\right] \mathbb{E}\left[\left|D_{x_{1}} F_{k}\right|^{2}\left|D_{x_{2}} F_{k}\right|^{2}\right]+\mathbb{E}\left[\left|D_{x_{1}, x_{3}}^{2} F_{l}\right|^{2}\left|D_{x_{2}, x_{3}}^{2} F_{l}\right|^{2}\right] \mathbb{E}\left[\left|D_{x_{1}} F_{k}\right|^{2}\left|D_{x_{2}} F_{l}\right|^{2}\right] \\
& \left.+\mathbb{E}\left[\left|D_{x_{1}, x_{3}}^{2} F_{l}\right|^{2}\left|D_{x_{2}, x_{3}}^{2} F_{l}\right|^{2}\right] \mathbb{E}\left[\left|D_{x_{1}} F_{l}\right|^{2}\left|D_{x_{2}} F_{k}\right|^{2}\right]+\mathbb{E}\left[\left|D_{x_{1}, x_{3}}^{2} F_{l}\right|^{2}\left|D_{x_{2}, x_{3}}^{2} F_{l}\right|^{2}\right] \mathbb{E}\left[\left|D_{x_{1}} F_{l}\right|^{2}\left|D_{x_{2}} F_{l}\right|^{2}\right]\right)^{1 / 2} \\
& +\int_{\mathbb{X}^{3}}\left(\mathbb{E}\left[\left|D_{x_{1}, x_{3}}^{2} F_{k}\right|^{2}\left|D_{x_{2}, x_{3}}^{2} F_{k}\right|^{2}\right]+\mathbb{E}\left[\left|D_{x_{1}, x_{3}}^{2} F_{k}\right|^{2}\left|D_{x_{2}, x_{3}}^{2} F_{l}\right|^{2}\right]\right. \\
& \left.\left.+\mathbb{E}\left[\left|D_{x_{1}, x_{3}}^{2} F_{l}\right|^{2}\left|D_{x_{2}, x_{3}}^{2} F_{k}\right|^{2}\right]+\mathbb{E}\left[\left|D_{x_{1}, x_{3}}^{2} F_{l}\right|^{2}\left|D_{x_{2}, x_{3}}^{2} F_{l}\right|^{2}\right]\right) \mu^{\otimes 3}(\mathrm{~d} \mathbf{x})\right)^{1 / 2} \\
& \leq\left[\int_{\mathbb{X}^{3}}\left(4\left(\mathbb{E}\left[\left|D_{x_{1}, x_{3}}^{2} F_{k}\right|^{2}\left|D_{x_{2}, x_{3}}^{2} F_{k}\right|^{2}\right]\right)^{1 / 2}\left(\mathbb{E}\left[\left|D_{x_{1}} F_{k}\right|^{2}\left|D_{x_{2}} F_{k}\right|^{2}\right]\right)^{1 / 2}+\mathbb{E}\left[\left|D_{x_{1}, x_{3}}^{2} F_{k}\right|^{2}\left|D_{x_{2}, x_{3}}^{2} F_{k}\right|^{2}\right]\right) \mu^{\otimes 3}(\mathrm{~d} \mathbf{x})\right. \\
& +\int_{\mathbb{X}^{3}}\left(4\left(\mathbb{E}\left[\left|D_{x_{1}, x_{3}}^{2} F_{l}\right|^{2}\left|D_{x_{2}, x_{3}}^{2} F_{l}\right|^{2}\right]\right)^{1 / 2}\left(\mathbb{E}\left[\left|D_{x_{1}} F_{l}\right|^{2}\left|D_{x_{2}} F_{l}\right|^{2}\right]\right)^{1 / 2}+\mathbb{E}\left[\left|D_{x_{1}, x_{3}}^{2} F_{l}\right|^{2}\left|D_{x_{2}, x_{3}}^{2} F_{l}\right|^{2}\right]\right) \mu^{\otimes 3}(\mathrm{~d} \mathbf{x}) \\
& +\int_{\mathbb{X}^{3}}\left(4\left(\mathbb{E}\left[\left|D_{x_{1}, x_{3}}^{2} F_{k}\right|^{2}\left|D_{x_{2}, x_{3}}^{2} F_{l}\right|^{2}\right]\right)^{1 / 2}\left(\mathbb{E}\left[\left|D_{x_{1}} F_{k}\right|^{2}\left|D_{x_{2}} F_{l}\right|^{2}\right]\right)^{1 / 2}+\mathbb{E}\left[\left|D_{x_{1}, x_{3}}^{2} F_{k}\right|^{2}\left|D_{x_{2}, x_{3}}^{2} F_{l}\right|^{2}\right]\right) \mu^{\otimes 3}(\mathrm{~d} \mathbf{x}) \\
& +\int_{\mathbb{X}^{3}}\left(4\left(\mathbb{E}\left[\left|D_{x_{1}, x_{3}}^{2} F_{l}\right|^{2}\left|D_{x_{2}, x_{3}}^{2} F_{k}\right|^{2}\right]\right)^{1 / 2}\left(\mathbb{E}\left[\left|D_{x_{1}} F_{l}\right|^{2}\left|D_{x_{2}} F_{k}\right|^{2}\right]\right)^{1 / 2}+\mathbb{E}\left[\left|D_{x_{1}, x_{3}}^{2} F_{l}\right|^{2}\left|D_{x_{2}, x_{3}}^{2} F_{k}\right|^{2}\right]\right) \mu^{\otimes 3}(\mathrm{~d} \mathbf{x}) \\
& +4 \int_{\mathbb{X}^{3}}\left(\mathbb{E}\left[\left|D_{x_{1}, x_{3}}^{2} F_{k}\right|^{2}\left|D_{x_{2}, x_{3}}^{2} F_{k}\right|^{2}\right]\right)^{1 / 2}\left(\mathbb{E}\left[\left|D_{x_{1}} F_{k}\right|^{2}\left|D_{x_{2}} F_{l}\right|^{2}\right]\right)^{1 / 2} \mu^{\otimes 3}(\mathrm{~d} \mathbf{x}) \\
& +4 \int_{\mathbb{X}^{3}}\left(\mathbb{E}\left[\left|D_{x_{1}, x_{3}}^{2} F_{k}\right|^{2}\left|D_{x_{2}, x_{3}}^{2} F_{k}\right|^{2}\right]\right)^{1 / 2}\left(\mathbb{E}\left[\left|D_{x_{1}} F_{l}\right|^{2}\left|D_{x_{2}} F_{k}\right|^{2}\right]\right)^{1 / 2} \mu^{\otimes 3}(\mathrm{~d} \mathbf{x}) \\
& +4 \int_{\mathbb{X}^{3}}\left(\mathbb{E}\left[\left|D_{x_{1}, x_{3}}^{2} F_{k}\right|^{2}\left|D_{x_{2}, x_{3}}^{2} F_{k}\right|^{2}\right]\right)^{1 / 2}\left(\mathbb{E}\left[\left|D_{x_{1}} F_{l}\right|^{2}\left|D_{x_{2}} F_{l}\right|^{2}\right]\right)^{1 / 2} \mu^{\otimes 3}(\mathrm{~d} \mathbf{x}) \\
& +4 \int_{\mathbb{X}^{3}}\left(\mathbb{E}\left[\left|D_{x_{1}, x_{3}}^{2} F_{k}\right|^{2}\left|D_{x_{2}, x_{3}}^{2} F_{l}\right|^{2}\right]\right)^{1 / 2}\left(\mathbb{E}\left[\left|D_{x_{1}} F_{k}\right|^{2}\left|D_{x_{2}} F_{k}\right|^{2}\right]\right)^{1 / 2} \mu^{\otimes 3}(\mathrm{~d} \mathbf{x}) \\
& +4 \int_{\mathbb{X}^{3}}\left(\mathbb{E}\left[\left|D_{x_{1}, x_{3}}^{2} F_{k}\right|^{2}\left|D_{x_{2}, x_{3}}^{2} F_{l}\right|^{2}\right]\right)^{1 / 2}\left(\mathbb{E}\left[\left|D_{x_{1}} F_{l}\right|^{2}\left|D_{x_{2}} F_{k}\right|^{2}\right]\right)^{1 / 2} \mu^{\otimes 3}(\mathrm{~d} \mathbf{x}) \\
& +4 \int_{\mathbb{X}^{3}}\left(\mathbb{E}\left[\left|D_{x_{1}, x_{3}}^{2} F_{k}\right|^{2}\left|D_{x_{2}, x_{3}}^{2} F_{l}\right|^{2}\right]\right)^{1 / 2}\left(\mathbb{E}\left[\left|D_{x_{1}} F_{l}\right|^{2}\left|D_{x_{2}} F_{l}\right|^{2}\right]\right)^{1 / 2} \mu^{\otimes 3}(\mathrm{~d} \mathbf{x}) \\
& +4 \int_{\mathbb{X}^{3}}\left(\mathbb{E}\left[\left|D_{x_{1}, x_{3}}^{2} F_{l}\right|^{2}\left|D_{x_{2}, x_{3}}^{2} F_{k}\right|^{2}\right]\right)^{1 / 2}\left(\mathbb{E}\left[\left|D_{x_{1}} F_{k}\right|^{2}\left|D_{x_{2}} F_{k}\right|^{2}\right]\right)^{1 / 2} \mu^{\otimes 3}(\mathrm{~d} \mathbf{x}) \\
& +4 \int_{\mathbb{X}^{3}}\left(\mathbb{E}\left[\left|D_{x_{1}, x_{3}}^{2} F_{l}\right|^{2}\left|D_{x_{2}, x_{3}}^{2} F_{k}\right|^{2}\right]\right)^{1 / 2}\left(\mathbb{E}\left[\left|D_{x_{1}} F_{k}\right|^{2}\left|D_{x_{2}} F_{l}\right|^{2}\right]\right)^{1 / 2} \mu^{\otimes 3}(\mathrm{~d} \mathbf{x}) \\
& +4 \int_{\mathbb{X}^{3}}\left(\mathbb{E}\left[\left|D_{x_{1}, x_{3}}^{2} F_{l}\right|^{2}\left|D_{x_{2}, x_{3}}^{2} F_{k}\right|^{2}\right]\right)^{1 / 2}\left(\mathbb{E}\left[\left|D_{x_{1}} F_{l}\right|^{2}\left|D_{x_{2}} F_{l}\right|^{2}\right]\right)^{1 / 2} \mu^{\otimes 3}(\mathrm{~d} \mathbf{x}) \\
& +4 \int_{\mathbb{X}^{3}}\left(\mathbb{E}\left[\left|D_{x_{1}, x_{3}}^{2} F_{l}\right|^{2}\left|D_{x_{2}, x_{3}}^{2} F_{l}\right|^{2}\right]\right)^{1 / 2}\left(\mathbb{E}\left[\left|D_{x_{1}} F_{k}\right|^{2}\left|D_{x_{2}} F_{k}\right|^{2}\right]\right)^{1 / 2} \mu^{\otimes 3}(\mathrm{~d} \mathbf{x}) \\
& +4 \int_{\mathbb{X}^{3}}\left(\mathbb{E}\left[\left|D_{x_{1}, x_{3}}^{2} F_{l}\right|^{2}\left|D_{x_{2}, x_{3}}^{2} F_{l}\right|^{2}\right]\right)^{1 / 2}\left(\mathbb{E}\left[\left|D_{x_{1}} F_{k}\right|^{2}\left|D_{x_{2}} F_{l}\right|^{2}\right]\right)^{1 / 2} \mu^{\otimes 3}(\mathrm{~d} \mathbf{x}) \\
& \left.+4 \int_{\mathbb{X}^{3}}\left(\mathbb{E}\left[\left|D_{x_{1}, x_{3}}^{2} F_{l}\right|^{2}\left|D_{x_{2}, x_{3}}^{2} F_{l}\right|^{2}\right]\right)^{1 / 2}\left(\mathbb{E}\left[\left|D_{x_{1}} F_{l}\right|^{2}\left|D_{x_{2}} F_{k}\right|^{2}\right]\right)^{1 / 2} \mu^{\otimes 3}(\mathrm{~d} \mathbf{x})\right]^{1 / 2} .
\end{aligned}
$$

Finally, by (5.30), using firstly again the inequality $(a+b)^{1 / 2} \leq a^{1 / 2}+b^{1 / 2}, a, b \geq 0$, and secondly 
the identities (5.11), we have

$$
\begin{aligned}
\gamma_{1}\left(\frac{F_{k}-F_{l}}{\sqrt{2}}\right) & \leq \gamma_{1}\left(F_{k}\right)+\gamma_{1}\left(F_{l}\right)+\gamma\left(F_{k}, F_{l}, F_{k}, F_{l}\right)+\gamma\left(F_{l}, F_{k}, F_{l}, F_{k}\right) \\
& +\lambda\left(F_{k}, F_{k}, F_{k}, F_{l}\right)+\lambda\left(F_{k}, F_{k}, F_{l}, F_{k}\right)+\lambda\left(F_{k}, F_{k}, F_{l}, F_{l}\right)+\lambda\left(F_{k}, F_{l}, F_{k}, F_{k}\right) \\
& +\lambda\left(F_{k}, F_{l}, F_{l}, F_{k}\right)+\lambda\left(F_{k}, F_{l}, F_{l}, F_{l}\right)+\lambda\left(F_{l}, F_{k}, F_{k}, F_{k}\right)+\lambda\left(F_{l}, F_{k}, F_{k}, F_{l}\right) \\
& +\lambda\left(F_{l}, F_{k}, F_{l}, F_{l}\right)+\lambda\left(F_{l}, F_{l}, F_{k}, F_{k}\right)+\lambda\left(F_{l}, F_{l}, F_{k}, F_{l}\right)+\lambda\left(F_{l}, F_{l}, F_{l}, F_{k}\right) \\
& =\gamma_{1}\left(F_{k}\right)+\gamma_{1}\left(F_{l}\right)+2 \gamma\left(F_{k}, F_{l}, F_{k}, F_{l}\right)+2 \lambda\left(F_{k}, F_{k}, F_{k}, F_{l}\right) \\
& +\lambda\left(F_{k}, F_{k}, F_{l}, F_{l}\right)+2 \lambda\left(F_{l}, F_{k}, F_{k}, F_{k}\right)+2 \lambda\left(F_{k}, F_{l}, F_{l}, F_{k}\right)+2 \lambda\left(F_{k}, F_{l}, F_{l}, F_{l}\right) \\
& +\lambda\left(F_{l}, F_{l}, F_{k}, F_{k}\right)+2 \lambda\left(F_{l}, F_{l}, F_{l}, F_{k}\right) .
\end{aligned}
$$

Therefore (5.29) follows by the assumptions (5.13) (with $i=1$ ), (5.15) and (5.16).

By Lemma 5.4, assumption (5.12) and Lévy's continuity theorem, the sequence $\left\{F_{n}\right\}_{n \geq 1}$ converges in law to $Z$. At last, the claim follows by the Ibragimov and Lifshits criterion.

Acknowledgments We would like to thank Matthias Schulte for useful discussions about his paper [8], and the anonymous referees for a very careful reading of the article.

\section{References}

[1] L. Ambrosio, N. Fusco and D. Pallara Functions of bounded variations and free discontinuity problems. Oxford University Press, New York, 2000.

[2] E. Azmoodeh and I. Nourdin. Almost sure limit theorems on Wiener chaos: the non-central case. Electronic Communications in Probability, 24: 1-12, 2019.

[3] B. Bercu, I. Nourdin and M.S. Taqqu. Almost sure central limit theorems on the Wiener space. Stochastic Processes and their Applications, 120: 1607-1628, 2010.

[4] I. Berkes and E. Csáki. A universal result in almost sure central limit theory. Stochastic Processes and their Applications, 94: 105-134, 2001.

[5] G. Brosamler. An almost everywhere central limit theorem. Mathematical Proceedings of the Cambridge Philosophical Society, 104: 561-574, 1988. 
[6] J.R. Chazottes and S. Gouëzel. On almost sure versions of classical limit theorems for dynamical systems. Probability Theory and Related Fields, 138: 195-234, 2007.

[7] I.A. Ibragimov and M.A. Lifshits. On limit theorems of almost sure type. Theory of Probability and its Applications, 44: 254-272, 2000.

[8] R. Lachiéze-Rey, M. Schulte and J.E. Yukich. Normal approximation for stabilizing functionals. The Annals of Applied Probability, 29: 931-993, 2019.

[9] G. Last and M.D. Penrose. Poisson process Fock space representation, chaos expansion and covariance inequalities. Probability Theory and Related Fields, 150: 663-690, 2011.

[10] G. Last and M.D. Penrose. Lectures on the Poisson process. Cambridge University Press, Cambridge, 2017.

[11] G. Last, G. Peccati and M. Schulte. Normal approximation on Poisson spaces: Mehler's formula, second order Poincaré inequalities and stabilization. Probability Theory and Related Fields, 165: 667-723, 2014.

[12] P. Lévy. Théorie de l'addition des variables aléatoires. Gauthier-Villar, Paris, 1937.

[13] M. Peligrad and Q.M. Shao. A note on the almost sure central limit theorem for weakly dependent random variables. Statistics and Probability Letters, 22: 131-136, 1995.

[14] M.D. Penrose. Random geometric graphs. Oxford University Press, New York, 2003.

[15] M.D. Penrose. Laws of large numbers in stochastic geometry with statistical applications. Bernoulli, 13: 1124-1150, 2007.

[16] M.D. Penrose and J.E. Yukich. Central limit theorems for some graphs in computational geometry. The Annals of Applied Probability, 11: 1005-1041, 2001.

[17] M.D. Penrose and J.E. Yukich. Limit theory for point processes in manifolds. The Annals of Applied Probability, 23: 2161-2211, 2013.

[18] M. Reitzner, E. Spodarev and D. Zhaporazhets. Set reconstruction by Voronoi cells. Advances in Applied Probability, 44: 938-953, 2012.

[19] P. Schatte. On strong versions of the central limit theorem. Mathematische Nachrichten, 137: 249-256, 1988. 
[20] M. Schulte. A central limit theorem for the Poisson-Voronoi approximation. Advances in Applied Mathematics, 49: 285-306, 2012.

[21] C. Thäle and J.E. Yukich. Asymptotic theory for statistics of the Poisson-Voronoi approximation. Bernoulli, 22: 2372-2400, 2016.

[22] K.I. Yoshihara. Weakly dependent stochastic sequences and their applications. In Recent Topics on Weak and Strong Limit Theorems, Vol. XIV. Sanseido Co. Ltd, Chiyoda, 2004.

[23] J.E. Yukich. Surface order scaling in stochastic geometry. The Annals of Applied Probability, 25: 177-210, 2015.

[24] G. Zheng. Normal approximation and almost sure central limit theorem for non-symmetric Rademacher functionals. Stochastic Processes and their Applications, 127: 1622-1636, 2017. 